\title{
PAX6 maintains $\beta$ cell identity by repressing genes of alternative islet cell types
}

\author{
Avital Swisa, ${ }^{1}$ Dana Avrahami, ${ }^{1,2}$ Noa Eden, ${ }^{1}$ Jia Zhang, ${ }^{3}$ Eseye Feleke, ${ }^{2}$ Tehila Dahan, ${ }^{1}$ Yamit Cohen-Tayar, ${ }^{4}$ Miri Stolovich-Rain, ${ }^{1}$ \\ Klaus H. Kaestner, ${ }^{3}$ Benjamin Glaser, ${ }^{2}$ Ruth Ashery-Padan, ${ }^{4}$ and Yuval Dor ${ }^{1}$ \\ 'Department of Developmental Biology and Cancer Research, The Institute for Medical Research Israel-Canada, The Hebrew University-Hadassah Medical School, Jerusalem, Israel. ${ }^{2}$ Endocrinology and \\ Metabolism Service, Department of Internal Medicine, Hadassah-Hebrew University Medical Center, Jerusalem, Israel. ${ }^{3}$ Department of Genetics and Institute for Diabetes, Obesity, and Metabolism, \\ Perelman School of Medicine, University of Pennsylvania, Philadelphia, Pennsylvania, USA. ${ }^{4}$ Department of Human Molecular Cenetics and Biochemistry, Sackler Faculty of Medicine and Sagol School \\ of Neuroscience, Tel Aviv University, Tel Aviv, Israel.
}

Type 2 diabetes is thought to involve a compromised $\beta$ cell differentiation state, but the mechanisms underlying this dysfunction remain unclear. Here, we report a key role for the TF PAX6 in the maintenance of adult $\beta$ cell identity and function. PAX6 was downregulated in $\beta$ cells of diabetic $d b / d b$ mice and in WT mice treated with an insulin receptor antagonist, revealing metabolic control of expression. Deletion of Pax6 in $\beta$ cells of adult mice led to lethal hyperglycemia and ketosis that were attributed to loss of $\beta$ cell function and expansion of $\alpha$ cells. Lineage-tracing, transcriptome, and chromatin analyses showed that PAX6 is a direct activator of $\beta$ cell genes, thus maintaining mature $\beta$ cell function and identity. In parallel, we found that PAX6 binds promoters and enhancers to repress alternative islet cell genes including ghrelin, glucagon, and somatostatin. Chromatin analysis and shRNA-mediated gene suppression experiments indicated a similar function of PAX6 in human $\beta$ cells. We conclude that reduced expression of PAX6 in metabolically stressed $\beta$ cells may contribute to $\beta$ cell failure and $\alpha$ cell dysfunction in diabetes.

\section{Introduction}

Mature pancreatic $\beta$ cells are highly specialized for sensing blood glucose levels and secreting insulin. Extensive efforts have resulted in a detailed understanding of the transcriptional cascades leading to differentiation of $\beta$ cells from progenitor cells during embryonic development and their subsequent maturation (1-3). More recently, it has emerged that, even after differentiation, the maintenance of adult $\beta$ cell identity and function requires the continuous activity of multiple transcription factors (TFs) (4-6). Importantly, some of these factors are sensitive to metabolic insults. For example, oxidative stress reduces the activity of the $\beta$ cell TFs PDX1, NKX6.1, and MAFA, suggesting a pathogenic mechanism for the development of diabetes (7). Perturbation of such factors by either genetic or environmental insults may result in $\beta$ cell death, or, alternatively, in loss of $\beta$ cell identity.

Interestingly, the loss of $\beta$ cell differentiation is often accompanied by acquisition of alternative cellular identities $(5,8,9)$, reflecting the retention of some developmental plasticity in differentiated $\beta$ cells. Typically, these alternative fates remain within the endocrine lineage. They may include, for example, a switch from expression of insulin to expression of glucagon or somatostatin, indicating that $\beta$ cell plasticity is largely confined to the islet program. The potential of metabolically stressed $\beta$ cells to dedifferen-

\section{Related Commentary: p. 94}

Conflict of interest: The authors have declared that no conflict of interest exists Submitted: April 11, 2016; Accepted: October 13, 2016

Reference information: / Clin Invest. 2017;127(1):230-243. doi:10.1172/JCl88015. tiate and then redifferentiate into non- $\beta$ cell fates was proposed as a novel mechanism underlying reversible $\beta$ cell failure in diabetes $(8,10-13)$. Plasticity between $\alpha$ and $\beta$ cell fates is supported by the remarkably similar epigenetic states of the 2 cell types (14). Other islet cell types also show such intraislet plasticity, and may, in some instances, reprogram into functional $\beta$ cells. For example, it was shown that near-total ablation of $\beta$ cells in mice results in the spontaneous conversion of some $\delta$ cells (15) or $\alpha$ cells (16) to functional $\beta$ cells, suggesting novel approaches for regenerative therapy in diabetes. Thus, the molecular mechanisms that govern the maintenance of adult islet cell identity are of great interest, with implications for the prevention of $\beta$ cell failure as well as expansion of $\beta$ cell mass in diabetes.

In this study, we focus on the role of the paired and homeodomain TF PAX6 in adult $\beta$ cells. PAX6 is crucial for the generation of neuronal lineages in the CNS including the cortex and retina, as well as the differentiation of non-neuronal lineages of the eye (17-19). This TF acts in these situations as both a transcriptional activator and repressor via complex gene regulatory networks that are only partly resolved (20-23). In the pancreas, PAX6 is required for normal islet development. In the absence of PAX6, the production of $\alpha$ cells and $\beta$ cells is greatly reduced; instead, there is a dramatic increase in the expression of ghrelin, a gut hormone normally expressed only transiently in the fetal pancreas. Thus, during development of the pancreas, PAX6 acts to direct the differentiation of endocrine-committed progenitor cells to correct fates. The molecular targets of PAX6 in mediating these developmental decisions are only partly understood (24-26). Notably, mice and humans heterozygous for PAX6 show defects in nervous system development as 
A

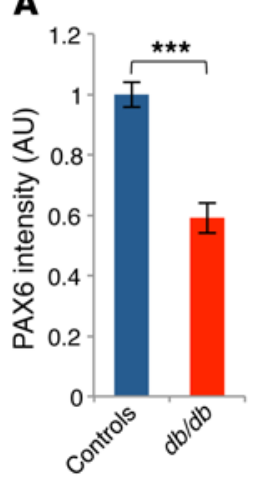

B

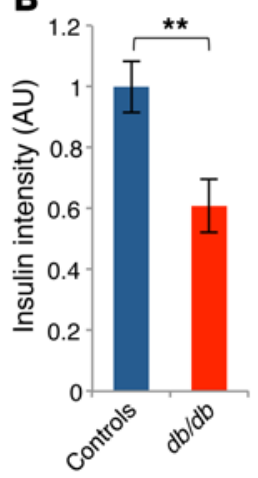

C

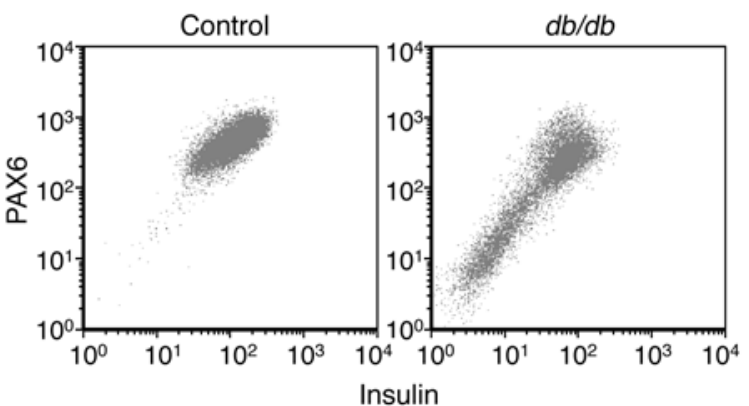

$\mathbf{F}$

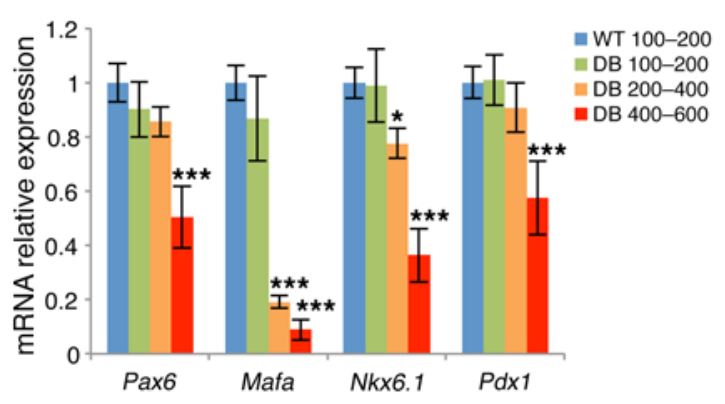

D

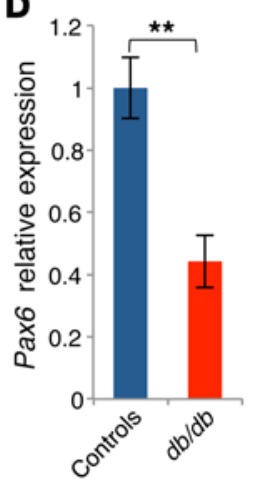

G

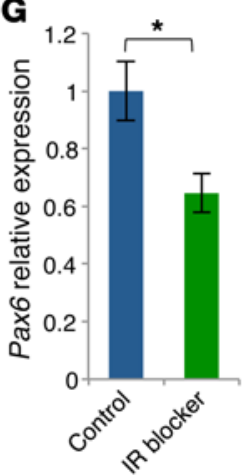

Figure 1. PAX6 expression in adult $\beta$ cells from $\boldsymbol{d b} \mathbf{b} / \mathbf{d} b$ mice and after treatment with an insulin receptor antagonist. (A) PAX6 protein levels in $\beta$ cells from $d b / d b$ mice and controls. Dissociated islet cells were costained for insulin and PAX6 and analyzed by flow cytometry. Graph shows the mean of PAX6 intensity in insulin ${ }^{+}$cells isolated from 3 control and $5 \mathrm{db} / \mathrm{db}$ mice at 3 months of age. (B) Mean insulin intensity for the same samples as in $\mathbf{A}$. (C) Representative plots of PAX6 versus insulin protein levels in the control and $d b / d b$ mice depicted in $\mathbf{A}$ and $\mathbf{B}$. PAX6 levels correlated with insulin levels in both control and $d b / d b$ mice. $d b / d b$ mice in panels A-C had, on average, glucose levels of $480 \mathrm{mg} / \mathrm{dl}$. (D) Pax6 mRNA levels in 3-month-old control and $d b / d b$ islets. $n=6$ animals per group. (E) Negative correlation between Pax6 mRNA and blood glucose levels in $d b / d b$ mice at different ages (4 weeks, 5-7 weeks, and 3 months, by Pearson's correlation test, $R=-0.9, P<0.05)$. (F) mRNA levels of Pax6, Mafa, Nkx6.1, and $P d x 1$ in the same $d b / d b$ (DB) mice as in E, averaged per blood glucose ranges (100-200, 200-400, and 400-600 mg/dl). $n=9,7,8$, and 7 mice, respectively. (G) Pax6 mRNA levels of islets isolated from WT mice treated with the insulin receptor (IR) antagonist S961 for 4 days. Average blood glucose level was $434 \mathrm{mg} / \mathrm{dl}$ in the treated group. $n=3$ per each group. ${ }^{*} P<0.05,{ }^{* *} P<0.01$, and ${ }^{* *} P<0.001$, by 2 -tailed Student's $t$ test.

well as perturbed glucose homeostasis (27-29), underscoring the importance of tight control over its expression level. In addition, a common variant in the $P A X 6$ gene was associated with a reduction in both PAX6 expression and insulin secretion (30).

In the adult pancreas, PAX6 is expressed in all hormone-producing cells, suggesting a general role in terminally differentiated endocrine cells $(24,25)$. In immortalized $\beta$ cell lines, PAX6 was shown to bind and activate the promoters of insulin and additional $\beta$ cell genes $(26,31)$. Deletion of Pax 6 in adult murine islet cells causes hyperglycemia, with reduced expression of insulin, glucagon, and somatostatin and upregulated expression of ghrelin (32). A more recent study showed that deletion of Pax 6 in adult $\alpha$ or $\beta$ cells induces ghrelin expression in mutant cells (33). However, the molecular mechanisms underlying these phenotypes were not studied.

Here, we report that PAX6 is downregulated in $\beta$ cells in 2 models of hyperglycemia and insulin resistance: $d b / d b$ mice, a model for type 2 diabetes, and WT mice treated with an insulin receptor antagonist. Deletion of Pax 6 in adult murine $\beta$ cells led to lethal hyperglycemia and ketosis due to loss of $\beta$ cell differentiation, combined with expansion of WT $\alpha$ cells. Genome-wide molecular analysis revealed that PAX6 is an essential direct acti- vator of key genes responsible for $\beta$ cell identity and function; in parallel, PAX6 binds promoters and enhancers of genes normally silenced in $\beta$ cells to directly repress alternative islet cell programs. We also provide evidence that these functions of PAX6 are conserved in human $\beta$ cells. The findings highlight the fragility of $\beta$ cell identity and establish PAX6 as a central determinant of adult $\beta$ cell differentiation state and function. We propose that downregulation of PAX6 in metabolically stressed $\beta$ cells may contribute to both $\beta$ cell failure and $\alpha$ cell dysfunction in diabetes.

\section{Results}

Reduced expression of PAX6 in $\beta$ cells of insulin-resistant, hyperglycemic mice. We determined PAX6 levels in hyperglycemic $d b / d b$ mice, a model for type 2 diabetes. FACS analysis showed that PAX6 protein levels were reduced by $40 \%$ in $\beta$ cells of diabetic $d b / d b$ mice compared with levels in controls (Figure 1A). The reduction in PAX6 protein levels mirrored the reduced insulin content in $\beta$ cells of $d b / d b$ mice (Figure 1B). Strikingly, PAX6 levels correlated with cellular insulin content in individual $\beta$ cells from both control and diabetic mice (Figure $1 \mathrm{C}$ ). These results suggest, but do not prove, that PAX6 is an important determinant of insulin content in 

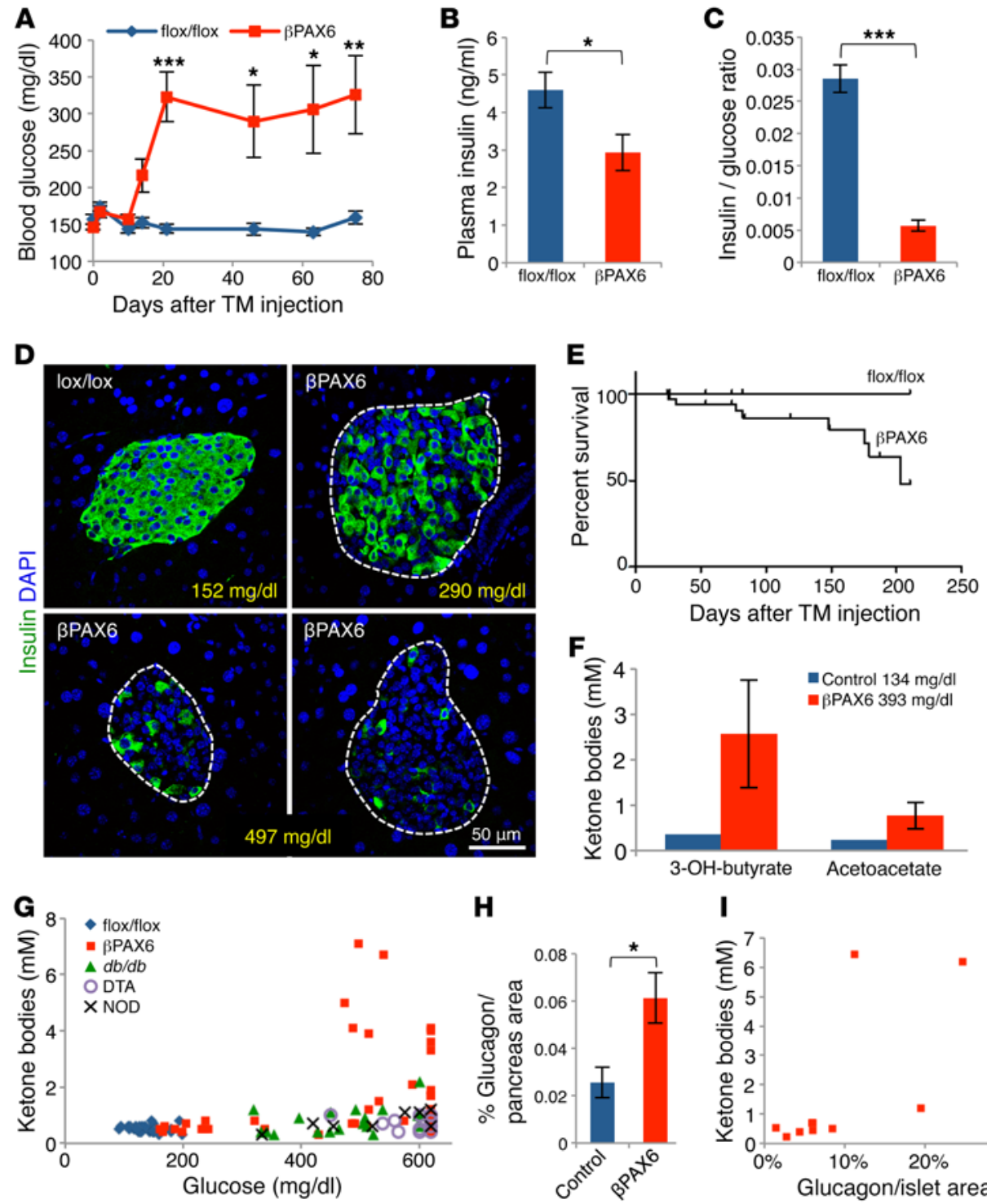

Figure 2. Hyperglycemia, hypoinsulinemia, hyperketonemia and loss of $\beta$ cells in $\beta$ PAX6 mice. (A) Blood glucose levels over time in Pax $6^{f / f l}$ (flox/flox) and $\beta P A X 6$ mice following tamoxifen (TM) injection. Tamoxifen was injected into mice at 6 weeks of age. $n=13$ flox/flox and $17 \beta$ PAX6 mice. (B) Plasma insulin levels in $\beta$ PAX6 and flox/flox mice at the age of 4 months and 2 months after tamoxifen injection. $n=5$ mice in each group. (C) Ratio of plasma insulin to blood glucose levels in the same mice as represented in B. (D) Representative insulin staining (green) of pancreatic sections from 1 flox/flox mouse and $2 \beta P A X 6$ mice. Blood glucose of each mouse is indicated. Note that despite a loss of insulin-stained cells, islets retained typical morphology and size. Original magnification, $\times 400$. (E) Survival of $\beta P A X 6$ mice. $n=13$ flox/flox and $35 \beta P A X 6$ mice. Graph shows the percentage of surviving mice up to 7 months following tamoxifen administration at 1 month of age. $P<0.05$, by log-rank (Mantel-Cox) test. (F) Ketone bodies in plasma of control and $\beta$ PAX6 mice $(n=6)$. Mice were injected with tamoxifen at 1 month of age and analyzed 3-5 months later. (C) Blood ketone bodies as a function of blood glucose levels in $\beta$ PAX6 mice and other models of murine diabetes. Note that the highest levels of ketone bodies were not always detected in the most hyperglycemic mice. (H) Quantification of $\alpha$ cell proportion of the total pancreatic area in $\beta$ PAX6 mice aged 3-6 months. Tamoxifen was injected at 1 month of age. $n=4$ flox $/$ flox and $7 \beta$ PAX6 mice. (I) Positive correlation between blood ketone bodies and the fraction of glucagon-stained area in $\beta P A X 6$ mice 3-6 months after tamoxifen injection ( $P<0.05$, by Spearman's correlation). Glucagon was measured and calculated from histological sections as the ratio between the area stained for glucagon and the total islet area. Each point represents 1 mouse. ${ }^{*} P<0.05,{ }^{* *} P<0.01$, and ${ }^{* * *} P<0.001$, by 2 -tailed Student's $t$ test.

adult $\beta$ cells and that its downregulation in diabetes may contribute to loss of insulin and $\beta$ cell dysfunction. This idea is particularly attractive, given the established genetic haploinsufficiency of PAX6 for glucose tolerance $(27,29,34)$. Consistent with the reduction in protein levels, Pax6 mRNA was reduced by approximately $55 \%$ in islets of diabetic $d b / d b$ mice (Figure 1D), indicating regulation at the level of transcription or mRNA stability. Importantly, measurements of Pax6 mRNA at various stages during the progression of $d b / d b$ mice to diabetes revealed that it was downregulated in severely hyperglycemic (400-600 $\mathrm{mg} / \mathrm{dl}$ glucose), but not in mildly hyperglycemic (200-400 $\mathrm{mg} / \mathrm{dl}$ ), mice (Figure 1E). A similar pattern was observed for other key $\beta$ cell TFs - Pdx1, Nkx6.1, and Mafa - although Mafa levels were reduced in much lower levels of hyperglycemia, indicating a higher sensitivity to glucose (Figure 1F and Supplemental Figure 1; supplemental material available online with this article; doi:10.1172/ JCI88015DS1). These findings suggest that PAX6 downregulation is probably not a primary driver of $\beta$ cell failure and diabetes, but rather a responder and potential contributor to glucotoxicity.

To further investigate the control of Pax6 expression in diabetes, we treated WT mice with the insulin receptor antagonist S961 (35). Four days of treatment led to severe hyperglycemia and resulted in a 40\% decrease in Pax6 mRNA levels compared with levels in controls (Figure 1G). These results indicate that downregulation of PAX6 in diabetes is not a peculiarity of $d b / d b$ mice, but rather a response to metabolic changes associated with insulin resistance and hyperglycemia. Further work is required to pinpoint the molecular mechanisms that control PAX6 expression in diabetes.

Efficient tamoxifen-induced deletion of Pax 6 in adult $\beta$ cells. To understand the function of PAX6 in mature $\beta$ cells, we deleted the gene in $\beta$ cells of adult mice. We generated MIPCreER Pax6 $6^{\text {f/ll }}$ Rosa26-LSL-YFP mice (referred to hereafter as $\beta$ PAX6 mice) with a conditional deletion of Pax6 and performed simultaneous genetic lineage tracing of $\beta$ cells. PAX6 was expressed in most $\beta$ cells in WT mice as well as in $\operatorname{Pax}^{\sharp / / l}$ and $\beta$ PAX 6 mice in the absence of tamoxifen, confirming tight control of Cre recombinase activity (Supplemental Figure 2). Injection of tamoxifen into $\beta$ PAX6 mice (2 doses of 
A
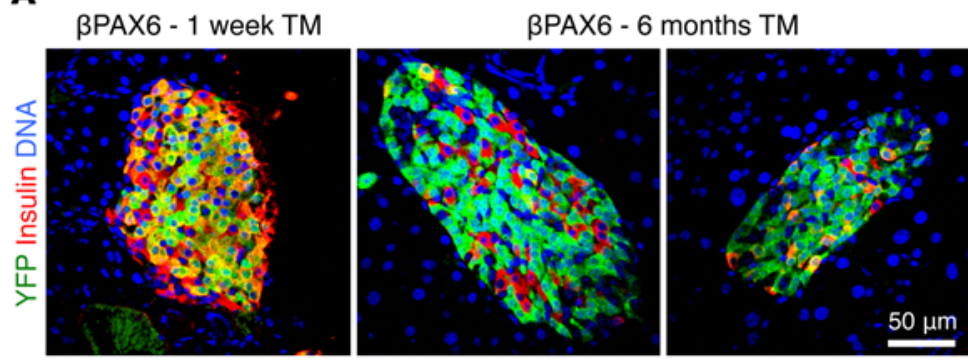

B
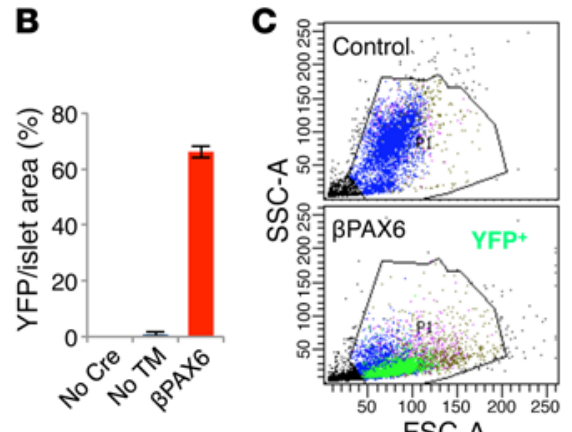

D

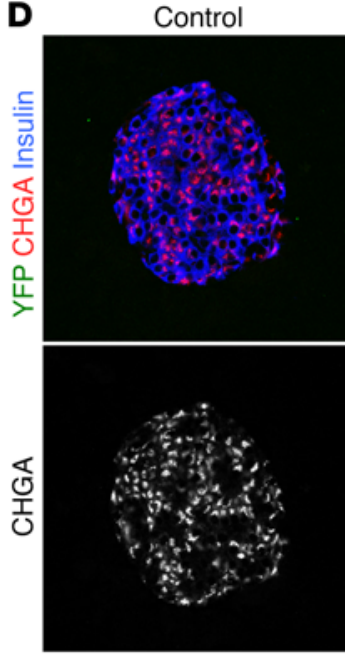

BPAX6 - 1 week

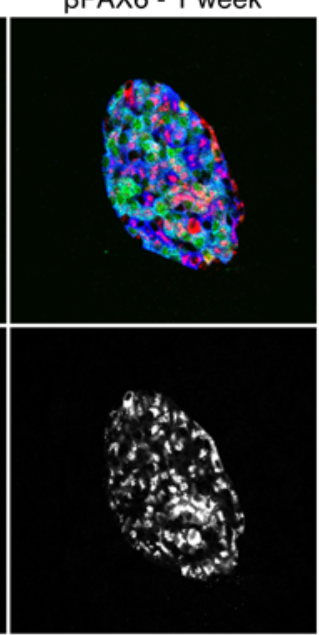

BPAX6 - 6 months
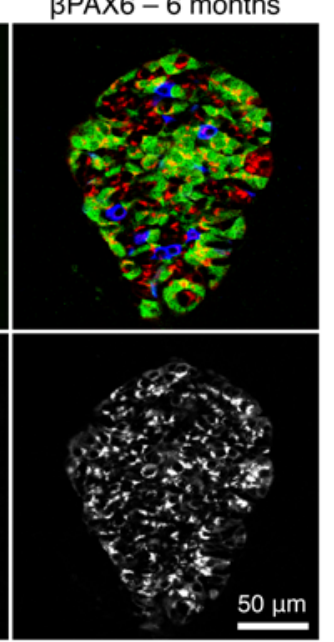

E

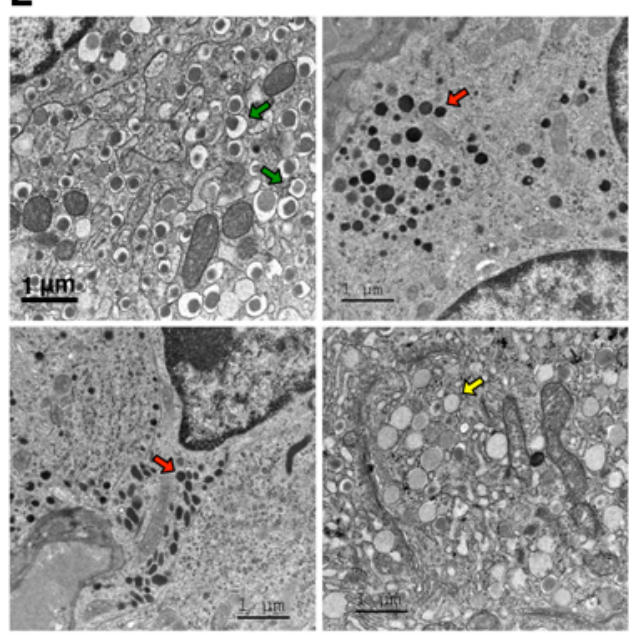

Figure 3. Lineage tracing of Pax6-deleted $\beta$ cells. (A) Loss of insulin in Pax6-deleted $\beta$ cells, labeled with YFP, after tamoxifen injection. Immunostaining for YFP (green), insulin (red), and DNA (blue). Note that most remaining insulin cells at 6 months were YFP- (i.e., had probably escaped deletion of Pax6). Mice were injected with tamoxifen at 2 months of age and sacrificed 1 week or 6 months later as indicated. Original magnification, $\times 400$. (B) Quantification

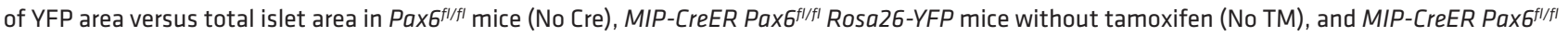
Rosa26-YFP mice 1 week after tamoxifen injection ( $\beta$ PAX6). $n=3$ mice in each group. (C) FACS analysis of sorted YFP $\beta$ cells 3 weeks after tamoxifen injection. $\beta P A X 6$ cells show a low side scatter (SSC) signal, probably indicating degranulation of $\beta$ cells. The control sample was from a Pax $6^{f / f f}$ (flox/flox) mouse. Analysis was performed on 4 samples of each genotype. FSC-A, forward scatter. (D) Pancreatic sections stained for chromogranin A (CHGA, red), YFP (green), and insulin (blue). Chromogranin was retained following long-term deletion of Pax6, suggesting retention of islet cell identity. Original magnification, $\times 400$. (E) Representative electron micrographs of pancreatic sections from a $\beta$ PAX6 mouse at the age of 3 months, 2 months after tamoxifen injection. Top left: normal insulin granules in WT islet (green arrow); top right: atypical cell type with dark granules (red arrows); bottom left: another atypical cell (red arrows); bottom right: $\beta$ cell with empty granules (yellow arrow). Samples from 3 flox/flox and $3 \beta$ PAX6 mice were imaged by electron microscopy. Original magnification, $\times 3,000$.

$8 \mathrm{mg}$ ) resulted in a rapid and efficient loss of PAX6 protein in more than $90 \%$ of $\beta$ cells as soon as 1 week after injection. PAX6 expression persisted in insulin cells in the periphery of the islet where $\alpha$ and $\delta$ cells are located, indicating $\beta$ cell-specific deletion of Pax6 (Supplemental Figure 2). We also sorted yellow fluorescent protein-positive $\left(\mathrm{YFP}^{+}\right) \beta$ cells from $\beta \mathrm{PAX} 6$ and control mice and measured the mRNA levels of Pax6 using quantitative reverse transcription PCR (qRT-PCR). One week after tamoxifen administration, Pax6 mRNA was reduced by $80 \%$ in $\beta$ cells isolated from $\beta$ PAX6 islets compared with levels in controls, as expected (Supplemental Figure 2).

Pax6 deletion in adult $\beta$ cells leads to progressive, lethal diabetes. Blood glucose levels started to rise 12 days after tamoxifen injection, and by 21 days, $\beta$ PAX 6 mice became severely hyperglycemic (Figure 2A). As expected with a primary defect in $\beta$ cells, hyperglycemia correlated with a reduction in plasma insulin levels in 3 PAX6 mice (Figure 2B) as well as a reduction of the insulin-to-glucose ratio (Figure 2C). Immunostaining of pancreatic sections revealed a near-complete loss of insulin expression in many $\beta$ PAX6 islets (Figure 2D). The number of remaining insulin ${ }^{+}$ cells varied between mice, probably due to a variation in recombination efficiency, and was correlated with the glycemic state of the mice (i.e., mice with fewer insulin ${ }^{+}$cells had more severe hyperglycemia, Figure 2D).

Interestingly, $50 \%$ of the $\beta$ PAX 6 mice died 1-7 months after tamoxifen injection (Figure 2E). This finding is difficult to explain by hyperglycemia alone, since multiple mouse models of diabetes, in our laboratory and elsewhere, survive well with similar or even higher levels of blood glucose $(36,37)$. Since ketoacidosis is known to be an acute cause of death in diabetes, we hypothesized that BPAX6 mice had elevated levels of ketone bodies in their blood. Indeed, we found very high levels of both 3-hydroxybutyrate and acetoacetate in the plasma of $\beta$ PAX6 mice compared with levels in control animals (Figure 2, F and G). By contrast, hyperglycemic mice expressing diphtheria toxin A (DTA) under an insulin- 
A

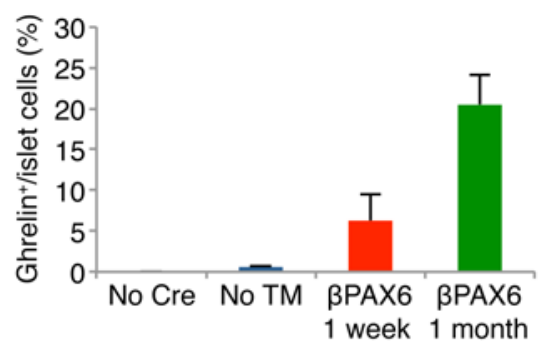

B

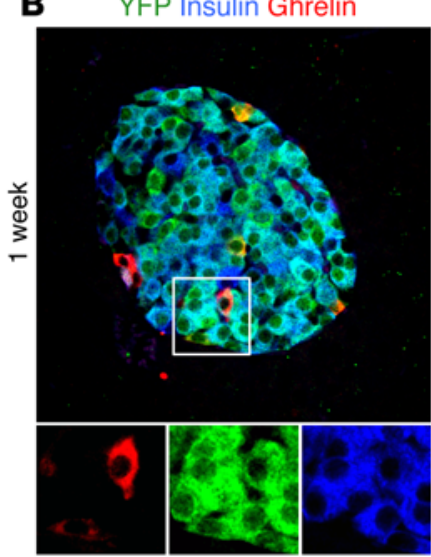

YFP Insulin Glucagon
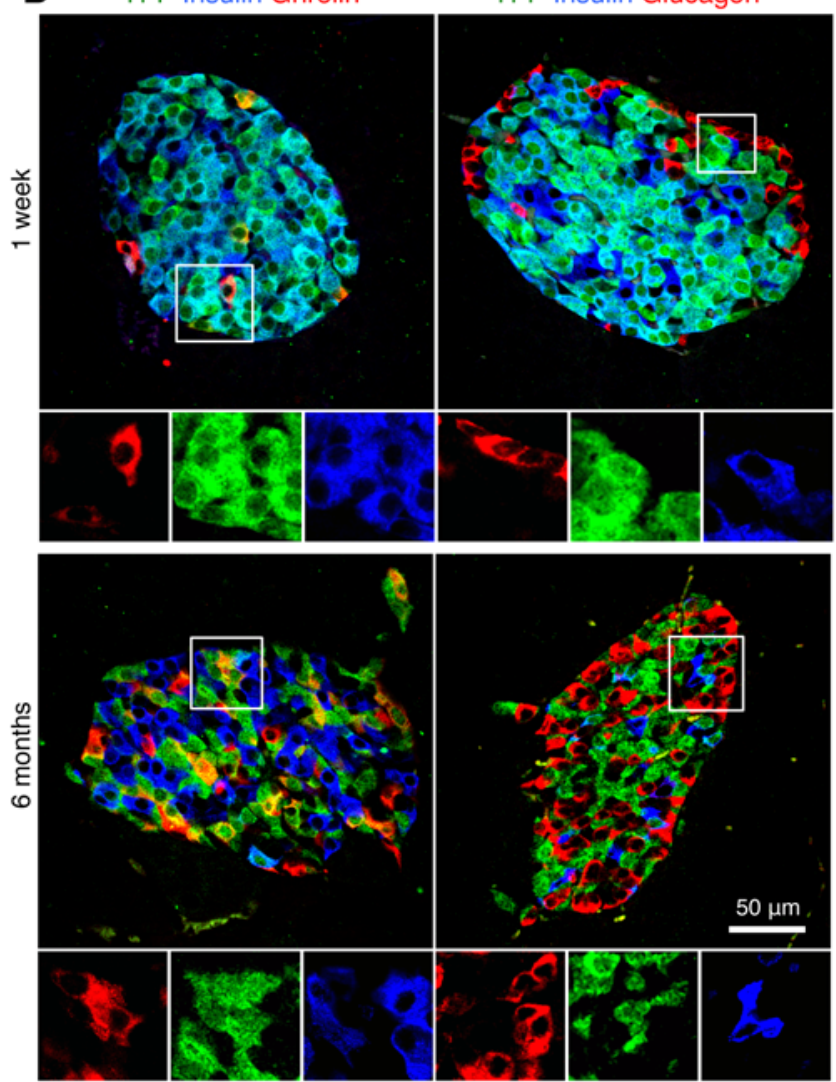

Figure 4. Origins of ghrelin- and glucagon-expressing cells in $\beta$ PAX6 mice. (A) Gradual increase in the fraction of islet cells expressing ghrelin in $\beta$ PAX6 mice compared with expression in controls (No Cre and No TM, as in Supplemental Figure 2). All mice were 3-4 months of age, 1 week after tamoxifen injection, except for mice in the "1 month" group, which were analyzed 1 month after tamoxifen administration (green bar). $n=3$, 6, 3, and 2 mice per group, respectively. (B) Pax6-deleted $\beta$ cells strongly expressed ghrelin, but not glucagon, as shown by costaining of YFP (green), insulin (blue), and ghrelin or glucagon (red). Islets shown were from $\beta$ PAX 6 mice 1 week or 6 months after tamoxifen injection. Original magnification, $\times 400$.

driven, rtTA-responsive promoter (insulin-rtTa TET-DTA mice) (36), $d b / d b$ mice, and NOD mice had minimal elevation of circulating ketones, supporting - though not proving - a link between ketones and death of diabetic $\beta$ PAX6 mice (Figure 2G).

BPAX6 mice with severe hyperglycemia or low levels of plasma insulin did not always show high blood ketone levels (Figure $2 \mathrm{G}$ and Supplemental Figure 3). Glucagon produced by $\alpha$ cells is a central driver of ketone formation, acting by inducing the expression of ketone biosynthesis enzymes in liver and adipose tissue
(38-40). To test whether hyperactivity of $\alpha$ cells could account for ketosis in $\beta$ PAX6, we measured plasma glucagon levels and the area stained by glucagon in the pancreas. Mutant mice with high ketone levels had higher levels of circulating glucagon than did controls (Supplemental Figure 3), and BPAX6 mice had a higher proportion of pancreas area stained by an anti-glucagon antibody (Figure 2H). Moreover, plasma ketone body levels correlated with the abundance of $\alpha$ cells in islets $(P<0.05$, Spearman's correlation test, Figure 2I, and see below). Thus, Pax 6 deletion in adult $\beta$ cells causes loss of $\beta$ cells, leading to hypoinsulinemia and hyperglycemia. In addition, this deletion results in hypersecretion of glucagon and severe ketosis, a feature rarely seen in mouse models of diabetes driven by insults to adult $\beta$ cells.

Pax6-deleted $\beta$ cells lose insulin but survive and maintain their endocrine identity. The loss of insulin staining in 3 PAX 6 mice could result from extensive $\beta$ cell death, or, alternatively, from loss of insulin expression and potentially other $\beta$ cell markers, as was found upon Foxo1 deletion (8). To distinguish between these possibilities, we monitored YFP expression, which serves as an indelible marker of $\beta$ cells following tamoxifen injection. YFP was present in nearly all $\beta$ cells 1 week after tamoxifen injection, and nearly all $\mathrm{YFP}^{+}$cells expressed insulin, indicating that YFP faithfully labeled $\beta$ cells (Figure 3, A and B). Examination of islets at later time points after Pax6 deletion revealed that $\mathrm{YFP}^{+}$cells remained abundant in islets but were insulin negative (Figure $3 \mathrm{~A}$ ). We found that the area occupied by $\mathrm{YFP}^{+}$cells was similar between BPAX6 and control MIP-CreER Rosa26-LSL-YFP mice (data not shown). $\beta$ PAX6 islets did not show massive staining for the cell death marker TUNEL, although we observed more TUNEL ${ }^{+}$islet cells in mutant mice than in controls (Supplemental Figure 4), suggesting the possibility that a lack of PAX6 compromises $\beta$ cell viability to some extent. While this remains to be further explored, we focused on the majority population of $\beta$ cells that survived Pax 6 deletion. Analysis by flow cytometry showed greatly diminished cytoplasmic insulin content in $\mathrm{YFP}^{+}$cells from $\beta$ PAX6 mice, manifested as decreased granularity (Figure $3 \mathrm{C}$ ). These results indicate that Pax 6 deletion in $\beta$ cells causes a gradual loss of insulin content, but that PAX6-deficient $\beta$ cells largely remain alive.

$\mathrm{YFP}^{+}$cells in $\beta$ PAX6 pancreata stained strongly for the endocrine marker chromogranin $\mathrm{A}$, indicating that PAX6-deficient $\beta$ cells retain their basic endocrine phenotype (Figure 3D and Supplemental Figure 5). This notion was supported by electron microscopic analysis of pancreatic sections from $\beta$ PAX 6 mice, which revealed an endocrine appearance of mutant islet cells, including secretory granules (Figure 3E). However, compared with control cells, BPAX6 islet cells were morphologically heterogeneous, with very few typical $\beta$ cells. Some cells had granules that lost the typical dense core appearance, while other cells had bigger, dark granules, not existing normally in islets and reminiscent of ghrelin-producing cells in the stomach (Figure 3E and Supplemental Figure 6) (41). Thus, Pax6 deletion causes a loss of insulin and ultrastructural features of $\beta$ cells and results in the acquisition of alternative endocrine cell morphologies.

Adult Pax6-deleted $\beta$ cells switch from insulin to ghrelin expression. To determine whether Pax6-deleted $\beta$ cells acquired a new endocrine identity, we stained $\beta$ PAX6 islets for all known islet hormones, including ghrelin, normally expressed only in fetal islets in mice. 
A

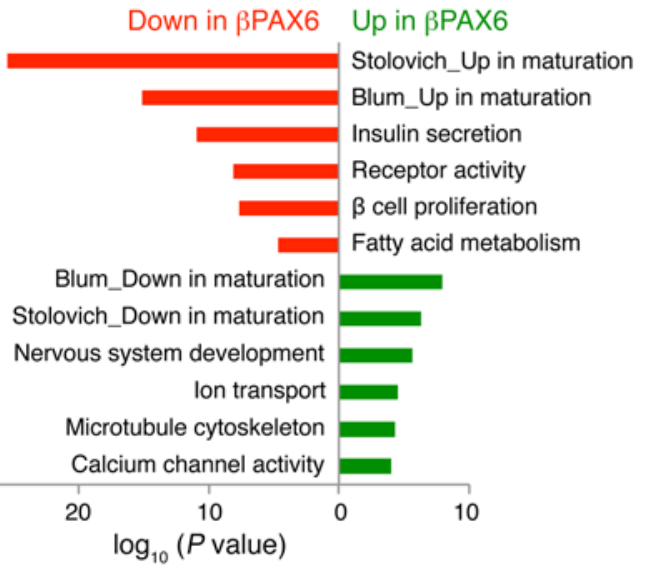

B

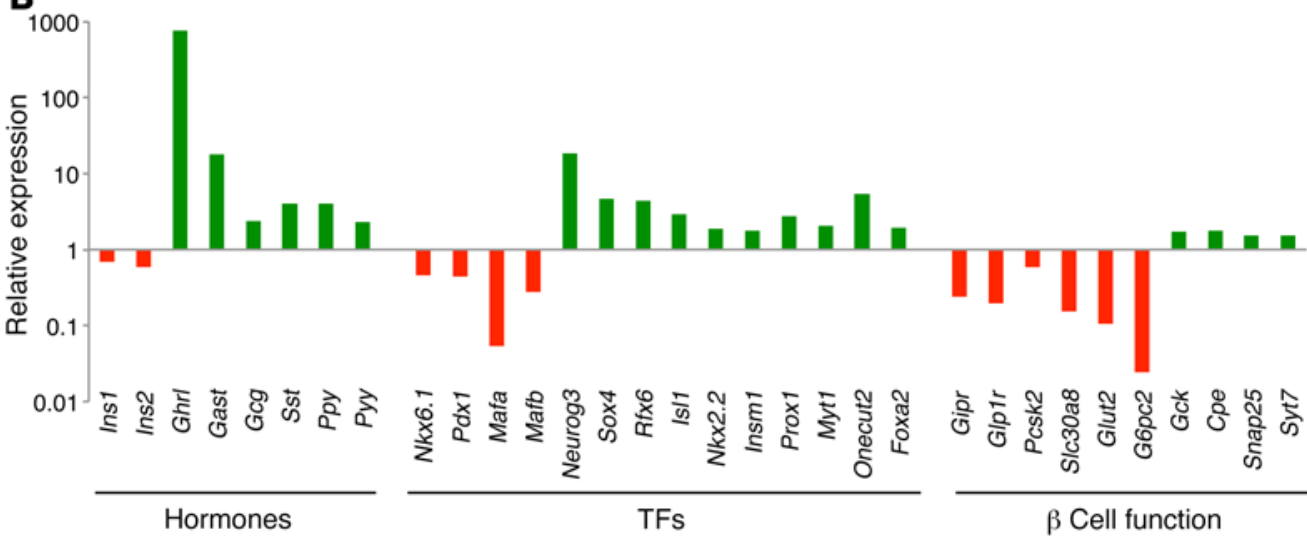

Figure 5. Transcriptomic analysis of PAX6-deficient $\beta$ cells. (A) Gene set enrichment analysis of sorted YFP+ cells from $\beta$ PAX6 mice $(n=3)$ compared with YFP controls $(n=4), 1$ week after tamoxifen injection. Cenes that were previously shown to be upregulated in mouse $\beta$ cell maturation ("Blum" adults vs. postnatal day 10 [ref. 76]; "Stolovich" postnatal day 35 vs. day 18 [Stolovich-Rain, unpublished observations]; see Supplemental Table 3) were downregulated in Pax6-deleted $\beta$ cells, as were gene sets of insulin secretion and $\beta$ cell proliferation. On the other hand, genes that were shown to be downregulated in maturation, as well as genes of nervous system development, were upregulated in Pax6-deleted $\beta$ cells. Interestingly, gene sets associated with ion transport and microtubule and calcium channel activity were also upregulated. (B) Fold change in mRNA of specific islet cell genes. Ghrelin was the top upregulated gene in Pax6-deleted $\beta$ cells. The FDR was below 0.05 for all genes, except Ins1 and Gcg $(P<0.05, \mathrm{FDR}<0.15)$. (C) Enrichment analysis of differentially expressed genes obtained in KO models of other TFs essential in cells. Nkx6.1 deletion in $\beta$ cells showed the closest similarity to Pax6 deletion.

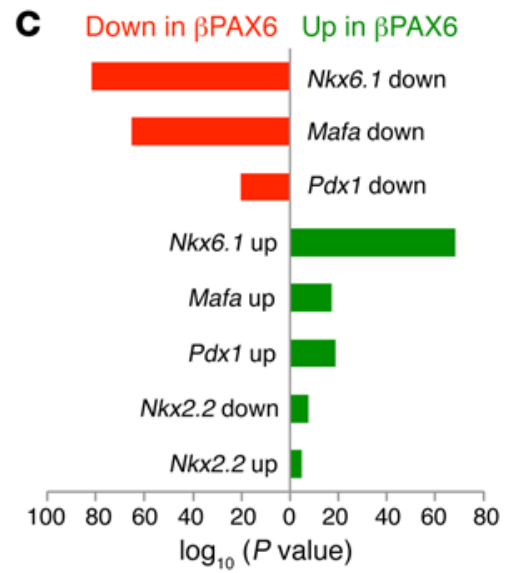

$4 \mathrm{~B}$ and Supplemental Figure 7). These results indicate that ghrelin expression occurs in $\beta$ cells following Pax6 deletion, while the expansion of $\alpha$ and $\delta$ cells occurs via a non-cellautonomous mechanism.

Transcriptome-wide changes in $\beta$ cell differentiation markers and TFs following Pax6 deletion. To further characterize the phenotype of $\beta$ cells following Pax 6 deletion, we determined their transcriptome. We sorted live $\mathrm{YFP}^{+}$cells from islets of control and $\beta P A X 6$ mice 1 week after tamoxifen administration and performed RNA sequencing. Gene set enrichment analysis showed that the most significant change following Pax6 deletion was a reduction in gene sets and genes, such as Slc2a2 (encoding GLUT2), G6pc2, and Slc3Oas (Figure 5, A and B, and Supplemental Tables 1 and 2), related to $\beta$ cell maturation and insulin secretion. At the protein level, we observed a dramatic reduction in GLUT2 levels by immunostaining (Supplemental Figure 8). Consistent with this finding, key $\beta$ cell TFs, including Pdx1, Mafa, and $N k x 6.1$, were downregulated (Figure 5B). The reduction of MAFA expression was evident even by immunostaining (Supplemental Figure 8).

Some gene sets associated with secretion or exocytosis,

One week after Pax6 deletion, we found no change in the abundance or distribution of cells expressing glucagon, somatostatin, and pancreatic polypeptide; however, we observed a striking appearance of cells expressing ghrelin (Figure 4A and Supplemental Figure 5). Three weeks after tamoxifen injection, $\beta$ PAX6 islets showed a further expansion of ghrelin staining as well as a dramatic increase in cells expressing glucagon or somatostatin (Supplemental Figure 5).

The abnormal abundance of cells expressing noninsulin hormones could emerge via a cell-autonomous mechanism (reprogramming of $\beta$ cells deficient for PAX6) or, alternatively, a non-cellautonomous mechanism (e.g., a paracrine effect of PAX6-deficient $\beta$ cells on neighboring cells). To distinguish between these possibilities, we costained for pancreatic hormones and the $\beta$ cell lineage marker YFP. Strikingly, ghrelin costained with YFP, indicating that ghrelin expression was initiated in cells that had expressed insulin at the time of tamoxifen injection. By contrast, glucagon, somatostatin, and pancreatic polypeptide did not costain with YFP (Figure e.g., those for nervous system development, ion transport, and calcium channels, were upregulated, in agreement with the observation that PAX6-deficient $\beta$ cells retained a secretory phenotype (Figure 5A and Supplemental Table 1). Surprisingly, mRNAs encoding all pancreatic hormones, including ghrelin, gastrin, glucagon, somatostatin, and pancreatic polypeptide, were upregulated in PAX6-deficient cells (even though only ghrelin could be detected at the protein level; Figure 4B and Supplemental Figure 5). We also observed upregulation of multiple TFs (Supplemental Table 2), including some known regulators of non- $\beta$ cell programs, such as Hhex (a direct activator of somatostatin transcription) (42).

The specific downregulation of the $\beta$ cell TFs Mafa, Nkx6.1, and $P d x 1$ suggests that PAX6 maintains $\beta$ cell identity through transcriptional activation of these genes. To examine this hypothesis, we compared transcriptomic changes that take place following deletion of each of these factors $(6,43,44)$ with the changes in $\beta$ PAX 6 cells. The genes that were up- or downregulated upon 
A

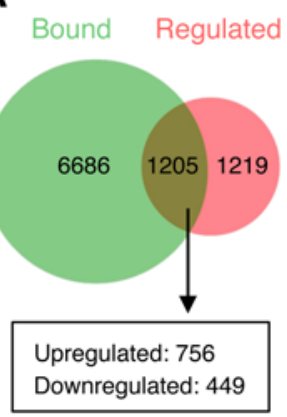

B

Top enriched motif, $P=1 \times 10^{-275}$

CTATATA ACC

\begin{tabular}{|c|c|c|}
\hline \multicolumn{3}{|c|}{ Motifs enriched in PAX6-binding sites } \\
\hline TF & Motif & $P$ value \\
\hline Ets & 'CCAACC年GAA & $1 \times 10^{-167}$ \\
\hline $\begin{array}{l}|s| 1 \mid \\
N k \times 6.1\end{array}$ & 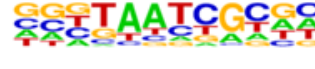 & $1 \times 10^{-125}$ \\
\hline Foxa2 & 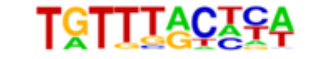 & $1 \times 10^{-117}$ \\
\hline $\mathrm{Nrf1}$ & TC수류 GGGCA & $1 \times 10^{-95}$ \\
\hline Neurod1 & AACAGATGGㅜㅜㅇ & $1 \times 10^{-92}$ \\
\hline
\end{tabular}

\begin{tabular}{|c|c|c|c|}
\hline \multirow{6}{*}{ 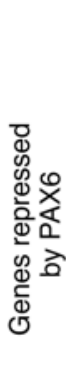 } & TF & Motif & $P$ value \\
\hline & $\begin{array}{l}P d \times 1 \\
(N k \times 6.1 / / s / 1)\end{array}$ & 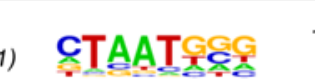 & $1 \times 10^{-31}$ \\
\hline & Foxa2 & TGTTTACي & $1 \times 10^{-21}$ \\
\hline & Neurod1 & 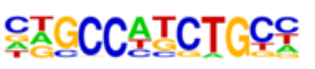 & $1 \times 10^{-19}$ \\
\hline & Pax6 & TTCACGCATGAG & $1 \times 10^{-18}$ \\
\hline & Pax2 & GCGTGACT & $1 \times 10^{-13}$ \\
\hline \multirow{4}{*}{ 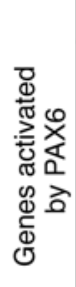 } & $N k \times 6.1$ & 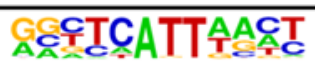 & $=1 \times 10^{-51}$ \\
\hline & Foxa2 & TGGGCAAACAA & $1 \times 10^{-31}$ \\
\hline & Neurod1 & AACAGCTGGC & $1 \times 10^{-18}$ \\
\hline & Pax3 & ATTAGTCACCC & $1 \times 10^{-15}$ \\
\hline
\end{tabular}

C

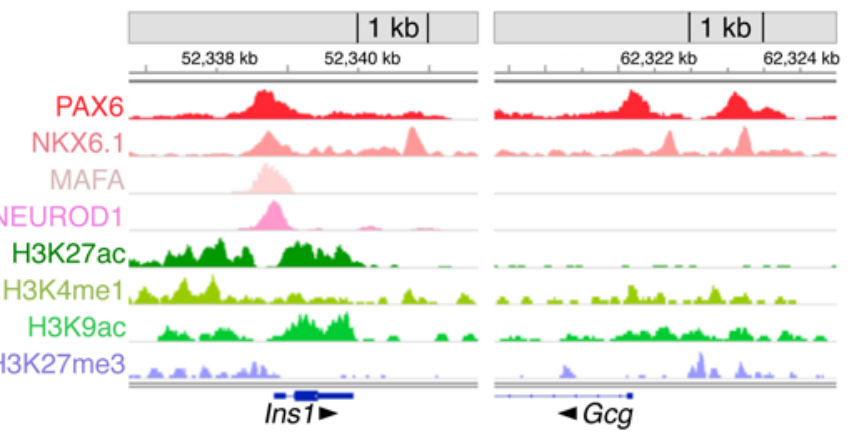

D

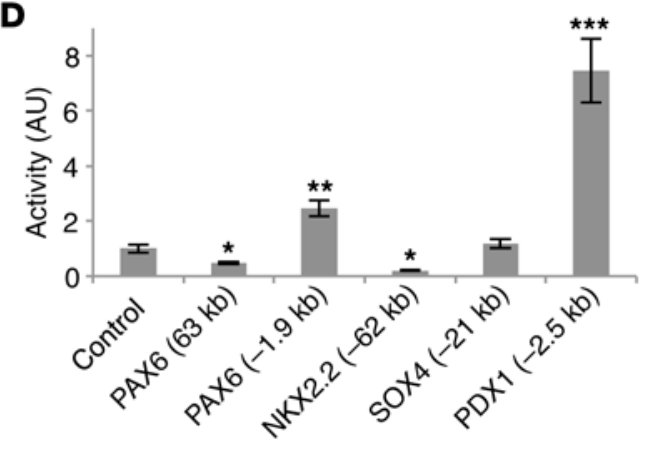

$\mathbf{E}$

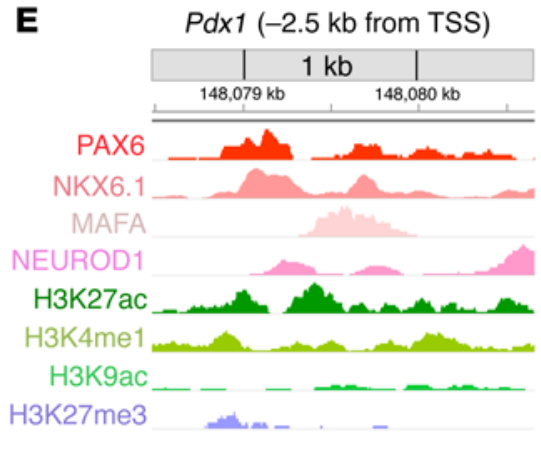

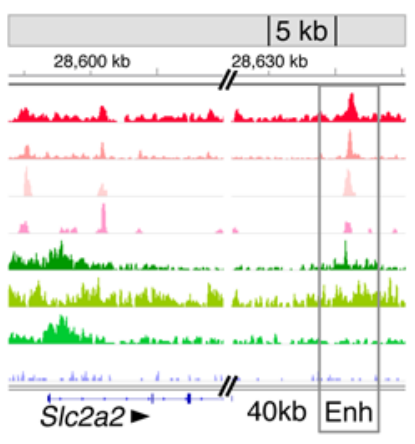

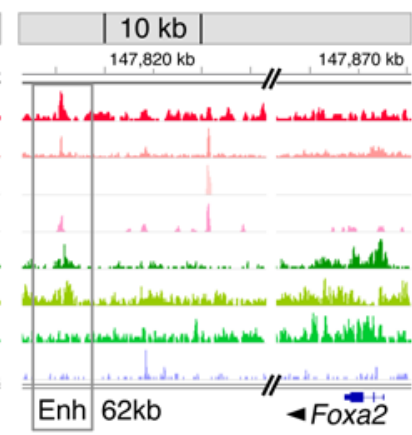

Figure 6. PAX6 ChIP-seq analysis in $\beta$ cells. (A) Venn diagram showing the overlap of genes regulated by PAX6 (from the RNA-seq analysis in $\beta$ PAX6 cells) with PAX6-bound genes (from ChIP-seq analysis in Min6 cells). (B) Top enriched motifs in all PAX6 peaks (left) and motifs differentially enriched in activated versus repressed genes in $\beta$ PAX6 mice (right). (C) Maps of representative key $\beta$ cell loci demonstrating the integration of PAX6 ChIP-seq data with other epigenomic data sets. Shown are PAX6-binding sites in the insulin and glucagon promoters and in Foxa2 and SIc2a2 (encoding GLUT2) enhancers (Enh, highlighted in rectangles; the distance from the transcription start site [TSS] is indicated). Note the active histone marks in insulin, Foxa2, and SIc2a2 versus the absence of active marks in the glucagon (Gcg) promoter. (D) Luciferase activity upon cloning of different PAX6-bound elements to a pGL4.23 luciferase reporter vector and transfection into Min6 cells. $n=3$ per element. ${ }^{*} P<0.05,{ }^{* *} P<0.01$, and ${ }^{* * *} P<0.001$, by 2-tailed Student's $t$ test. (E) Integrated map of histone marks and $\beta$ cell TFs binding at PAX6-bound regions found by luciferase assay (see Figure 6D) to have enhancer (Pdx1) and silencer (Nkx2.2) activity. Note the higher enrichment of H3K27ac in the Pdx1 enhancer region.

deletion of Nkx6.1, Mafa, and $P d x 1$ were significantly enriched in the differential $\beta$ PAX6 transcriptome (Figure 5C), consistent with the idea that the phenotype of $\beta$ PAX6 cells is at least partly caused by downregulation of these TFs. Interestingly, there was no overlap with genes altered upon $N k x 2.2$ deletion (45), a finding that was consistent with the lack of a major change in the expression of NKX2.2 itself (which was, in fact, mildly induced). NKX2.2 deficiency was previously shown to trigger massive expansion of ghrelin cells at the expense of cells (46). The fact that NKX2.2 and its targets were not significantly changed in $\beta$ PAX 6 cells suggests that PAX6 prevents ghrelin expression via an NKX2.2-independent mechanism. Overall, the transcriptomic changes observed strongly suggest that PAX6 is required to maintain the specific differentiation state of $\beta$ cells in adults and to repress alternative islet cell expression programs. However, PAX6 is not involved in maintaining the general endocrine identity of cells or in their survival. 
Table 1. Histone marks and binding of other $\beta$ cell TFs in genes bound and activated or bound and repressed by PAX6

\begin{tabular}{|c|c|c|c|c|c|c|c|c|c|}
\hline & НЗК27ас & НЗК4me1 & НЗК27me3 & НЗК9ас & MAFA & PDX1 & NEUROD1 & FOXA2 & NKX6.1 \\
\hline Genes activated by PAX6 & $41 \%$ & $21 \%$ & $7 \%$ & $3 \%$ & $6 \%$ & $28 \%$ & $14 \%$ & $17 \%$ & $21 \%$ \\
\hline Genes repressed by PAX6 & $26 \%$ & $23 \%$ & $15 \%$ & $2 \%$ & $2 \%$ & $22 \%$ & $11 \%$ & $13 \%$ & $15 \%$ \\
\hline
\end{tabular}

Numbers represent the percentage of PAX6 peaks that contain the indicated mark (see Supplemental Table 4 for details).

Direct transcriptional targets of PAX6 in $\beta$ cells. To study the mechanism of gene regulation by PAX6 in $\beta$ cells, we determined its direct targets using ChIP-sequencing (ChIP-seq) analysis in Min6 (mouse insulinoma) cells. PAX6 was physically associated with 9,789 genomic sites that were assigned to 7,891 genes (bound site and coding sequence located $<100 \mathrm{~kb}$ apart). PAX6 was bound to either the promoters of genes (11\% of bound sites, $1 \mathrm{~kb}$ from the transcription start site) or to distal sites. Among the PAX6-bound genes, $15 \%$ also showed significant changes in their steady-state mRNA levels in $\beta$ cells lacking PAX6. The preferential binding of PAX6 to genes that it regulates was statistically significant (PAX6 was bound to $50 \%$ of the genes that were regulated by it transcriptionally, but to only $32 \%$ of all nonregulated genes; $P<0.005$ by hypergeometric test), suggesting a direct regulation of these genes by PAX6 (Figure 6A).

While recognized as both a transcriptional activator and repressor in the nervous system, PAX6 has thus far been shown to be only an activator of gene expression in $\beta$ cells $(26,31)$. If this were the case, PAX6-bound genes in $\beta$ cells should be downregulated upon its deletion. Surprisingly, two-thirds (756 of 1,205) of the genes bound and regulated by PAX6 were in fact activated in Pax6-deficient $\beta$ cells (Figure 6A and Supplemental Table 4). This suggests that PAX6 functions as both a transcriptional activator and repressor in $\beta$ cells. Genes directly repressed by PAX6 in $\beta$ cells included the hormones glucagon, somatostatin (both of which were also upregulated nonautonomously in BPAX6 islets; Figure 4 and Supplemental Figure 7), and ghrelin and TFs including Pax 6 itself, Isl1, and Foxa2 (Supplemental Table 5).

How PAX6 represses transcription is not fully understood. Studies in the developing eye and various cell lines have shown that 2 different splice variants of PAX6 bind distinct DNA motifs, leading to either transcriptional activation or repression (47-50). We found both PAX6 splice variants to be expressed at high and equivalent levels in $\beta$ cells (data not shown), consistent with the idea that this mechanism is operative.

Our analysis of PAX6 ChIP-seq peaks for enrichment of recognition sequences identified a homeobox element highly similar to a known LIM homeobox (Lhx) and PAX4 motif, not previously recognized as a PAX6 motif, as the top enriched motif $\left(P=1 \times 10^{-275}\right)$ (Figure 6B). The motif was enriched in both upregulated and downregulated genes. In contrast, the known PAX6 motif (51) was enriched only among the genes upregulated by Pax6 deletion, suggesting that this motif is associated with PAX6-mediated transcriptional repression in $\beta$ cells. Additional motifs overrepresented in PAX6-bound regions resembled the known targets of other $\beta$ cell TFs such as ISL1, NKX6.1, FOXA2, NEUROD1, and PDX1, consistent with the notion that PAX6 cooperates with these factors in the regulation of $\beta$ cell expression programs.
Next, we asked whether other $\beta$ cell TFs and histone marks are differentially associated with PAX6-bound sites in the activated versus repressed genes. We overlapped the PAX6-bound sites with previously published whole-genome maps of monomethylated histone $\mathrm{H} 3$ at lysine 4 (H3K4me1, associated with enhancers); acetylated histone $\mathrm{H} 3$ at lysine 27 (H3K27ac, associated with active enhancers); acetylated histone $\mathrm{H} 3$ at lysine 9 (H3K9ac, associated with active promoters); and histone $\mathrm{H} 3$ lysine 27 trimethylation (H3K27me3, associated with repression) - all obtained from sorted mouse $\beta$ cells (52). In addition, we overlapped PAX6 ChIP-seq peaks with whole-genome chromatin-binding maps of MAFA, NKX6.1, PDX1, FOXA2, and NEUROD1 (6, 52, 53). Genes activated by PAX6 (downregulated in mutant $\beta$ cells) were enriched for the active enhancer mark H3K27ac, while genes repressed by PAX6 were enriched for the repressive mark H3K27me3, consistent with the idea that PAX6 functions as a direct repressor of these genes. The same pattern was observed in promoters and distal elements $(P<0.01)$ (Figure 6C and Table 1$)$. Interestingly, while key $\beta$ cell TFs (PDX1, MAFA, NEUROD1, FOXA2, and NKX6.1) bind both PAX6-activated and PAX6-repressed genes (Supplemental Table 4), the genes activated by PAX6 were more likely to be bound by these factors (Table 1). These results raise the possibility that the effect of PAX6 on a particular gene that it binds -i.e., activation or repression - is related to the number and identity of additional $\beta$ cell TFs occupying the same regulatory region.

Finally, to examine whether PAX6-bound sites can indeed function to enhance or silence gene expression, we selected several distal PAX6-bound elements associated with key islet genes and cloned them in front of a luciferase reporter gene driven by a minimal promoter. We transfected the reporters into Min6 cells and determined the potential regulatory activity of the cloned elements. Strikingly, we identified PAX6-bound sites that either activated or repressed luciferase expression, supporting the idea that PAX6 can function as a direct activator as well as a direct repressor of gene expression (Figure 6D). We then tested whether PAX6-bound regulatory elements that functioned in the luciferase assay as enhancers or silencers carried relevant histone marks in Min 6 cells. Indeed, the bound site associated with $N k x 2.2$ (which is repressed by PAX6 according to our RNA-seq analysis) showed a silencing activity in the reporter assay and was relatively depleted of the H3K27ac active enhancer mark; in contrast, the region associated with $P d x 1$ (activated by PAX6, according to our RNA-seq analysis) showed enhancer activity and was relatively enriched for H3K27ac (Figure 6E). These findings support the idea that PAX6 functions as a direct activator as well as a direct repressor of gene expression in $\beta$ cells.

A mechanism for indirect negative regulation of Neurog3 by PAX6 via direct repression of Foxa2. Multiple TFs were upregulated in mutant 
A
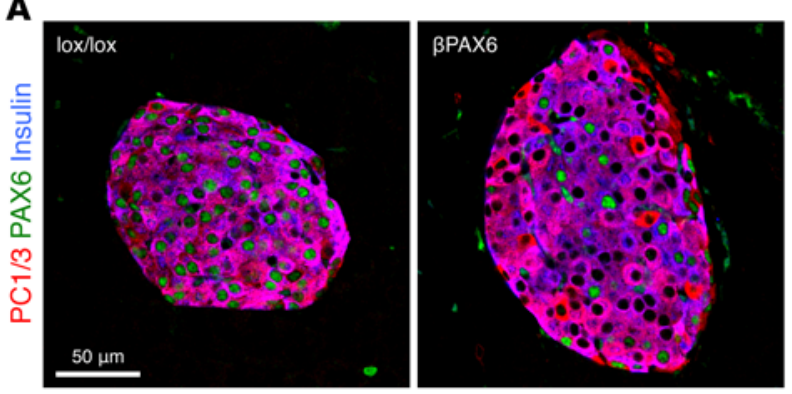

B

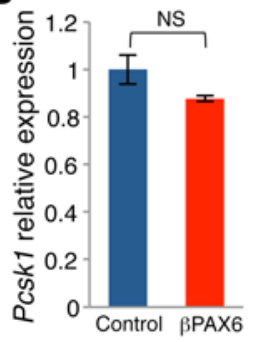

C
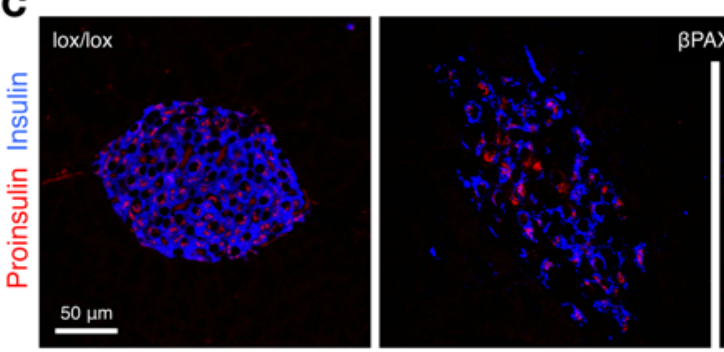

\section{PAX6}

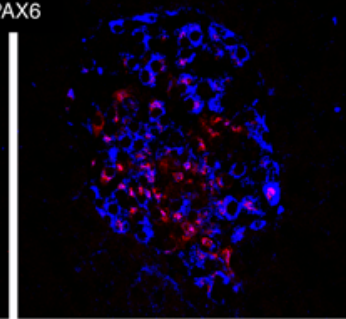

D

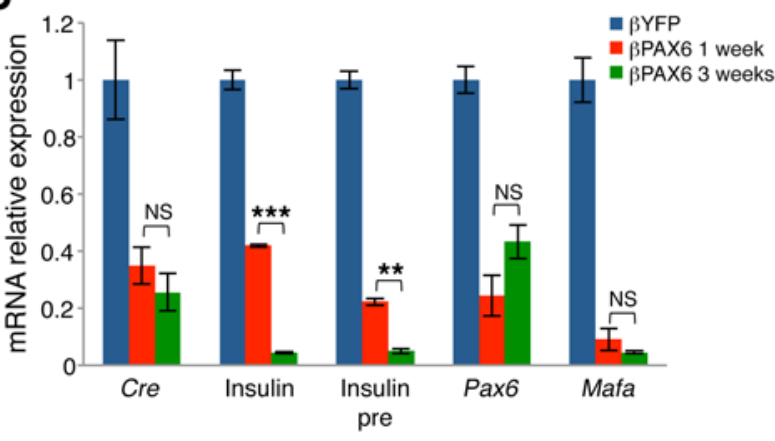

E

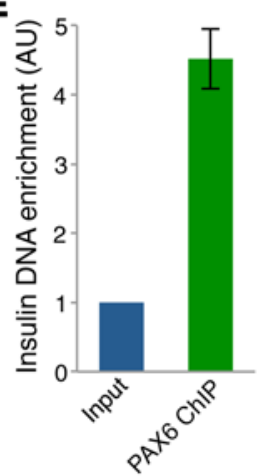

Figure 7. Regulation of insulin expression and processing by PAX6. (A) PC1/3 (encoded by Pcsk1) immunostaining was not reduced in Pax6-deleted $\beta$ cells. Staining for PC1/3 (red), PAX6 (green), and insulin (blue) in cells from 3-month-old mice, 1 week after tamoxifen administration. Original magnification, $\times 400$. (B) Pcsk1 mRNA level was not changed in sorted YFP $\beta$ cells from $\beta$ PAX 6 mice, 1 week after tamoxifen administration. $n=4$ control, $3 \beta$ PAX 6 mice. (C) Proinsulin staining of $\beta$ PAX 6 and control pancreatic section showed no accumulation of proinsulin (red) in insulin- (blue) cells. Mice were littermates of those represented in A. Original magnification, $\times 400$. (D) Transcriptional downregulation of insulin after Pax6 deletion. Reduced Ins1 mRNA, Ins1 pre-mRNA, Cre recombinase mRNA (driven by a transgenic insulin gene promoter), Pax6 mRNA, and Mafa mRNA after deletion of Pax6. Analysis was performed on sorted $\mathrm{YFP}^{+}$cells from control ( $\beta \mathrm{YFP}$ ) and $\beta$ PAX 6 mice injected with tamoxifen at 1 month of age and sacrificed 1 or 3 weeks later. $n=3$ for each group of mice. (E) ChIP with PAX6 antibody in Min6 cells showing enrichment for the Ins1 promoter $(n=2)$ compared with input. ${ }^{* *} P<0.01$ and ${ }^{* * *} P<0.001$, by 2 -tailed Student's $t$ test. $\beta$ cells (Figure $5 \mathrm{~B}$ ). Most notably, the fetal endocrine progenitor cell marker Neurog3 was elevated by 16-fold at the mRNA level, a finding that we confirmed by immunostaining (Supplemental Figure 8). PAX6 could directly repress the Neurog3 promoter or, alternatively, could affect a transcriptional regulator of Neurog3. Since the CHIPseq data did not reveal direct binding of PAX6 to the Neurog3 gene, we examined the expression levels of factors, including HES1, SOX9, ONECUT1, TCF2, and FOXA2, known to regulate Neurog3 expression during embryonic development $(54,55)$. Of these, Foxa 2 mRNA levels were 2-fold higher in PAX6-deficient $\beta$ cells (Figure 5B), suggesting a scenario in which PAX6 contributes to the silencing of Neurog 3 transcription in adult $\beta$ cells by repressing Foxa2. Consistent with this model, our ChIP-seq data revealed that PAX6 was bound to a Foxa2 enhancer (52) (Figure 6C). Thus, PAX6 represses Neurog3 expression in adult $\beta$ cells, and this probably occurs, at least in part, via repression of the Neurog3 regulator FOXA2. Whether derepressed Neurog3 in PAX6 mutants contributes to the phenotype of mutant cells remains to be determined via genetic loss-of-function experiments. Finally, Neurog3 is normally activated only transiently before shutting off its own promoter. The persistence of Neurog3 expression in PAX6-deficient $\beta$ cells raises the possibility that PAX6 participates in the autoregulation of Neurog3.

In summary, Pax 6 deletion in adult $\beta$ cells leads to the downregulation of $\beta$ cell-specific expression programs and their upstream TFs. In parallel, PAX6 deficiency derepresses alternative islet hormone genes and alternative islet TFs.

PAX6 directly regulates transcription from the insulin gene promoter. We performed experiments to better understand the loss of insulin in PAX6-deficient $\beta$ cells. Previous studies suggested that PAX6 regulates insulin by direct binding of the gene promoter as well as by regulating the expression of prohormone convertase $1 / 3$ (PC1/3), which is essential for insulin processing $(22,26,31,56$, 57). PAX6-deficient $\beta$ cells expressed PC1/3 strongly at both the mRNA and protein levels (Figure 7, A and B), indicating that $\mathrm{PC} 1 / 3$ is not activated by PAX6 in adult $\beta$ cells. In addition, no accumulation of proinsulin was observed in $\beta$ PAX 6 islets, again, arguing against a block in insulin processing. In fact, proinsulin levels were decreased in $\beta$ PAX6 islets (Figure 7C), suggesting that PAX6 regulates insulin expression at a stage prior to translation.

Next, we examined the effect of PAX6 on insulin transcription. We used the CreER transgene, driven by the mouse insulin promoter, as a reporter for insulin promoter activity. CreER mRNA was significantly reduced as early as 1 week after tamoxifen administration (Figure 7D), suggesting that PAX6 is needed for activation of the insulin promoter. Insulin mRNA levels decreased by only 2 -fold 1 week after tamoxifen administration but dropped further 2 weeks later. A similar pattern was observed for insulin pre-mRNA levels (Figure 7D). The different kinetics of decay of 
A

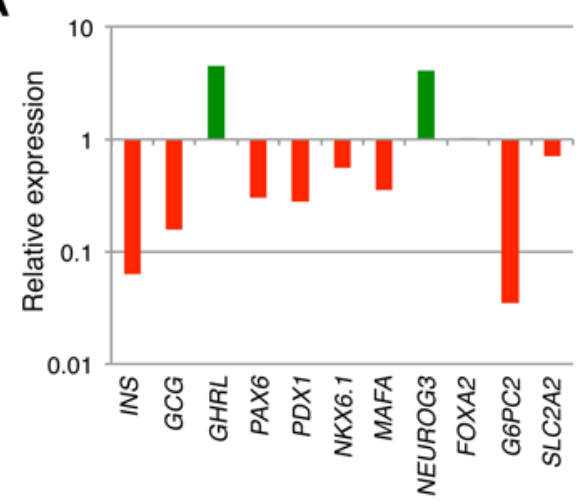

B

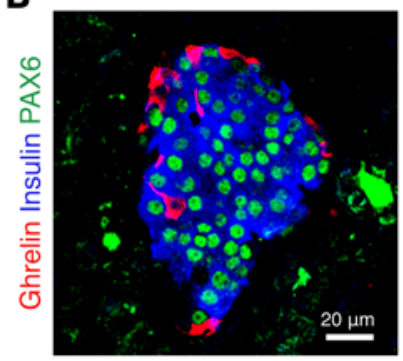

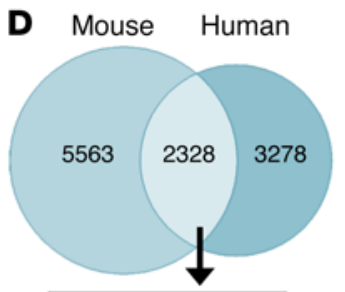

$\beta$ Cell genes

Calcium signaling

MODY genes

Insulin signaling T2D genes
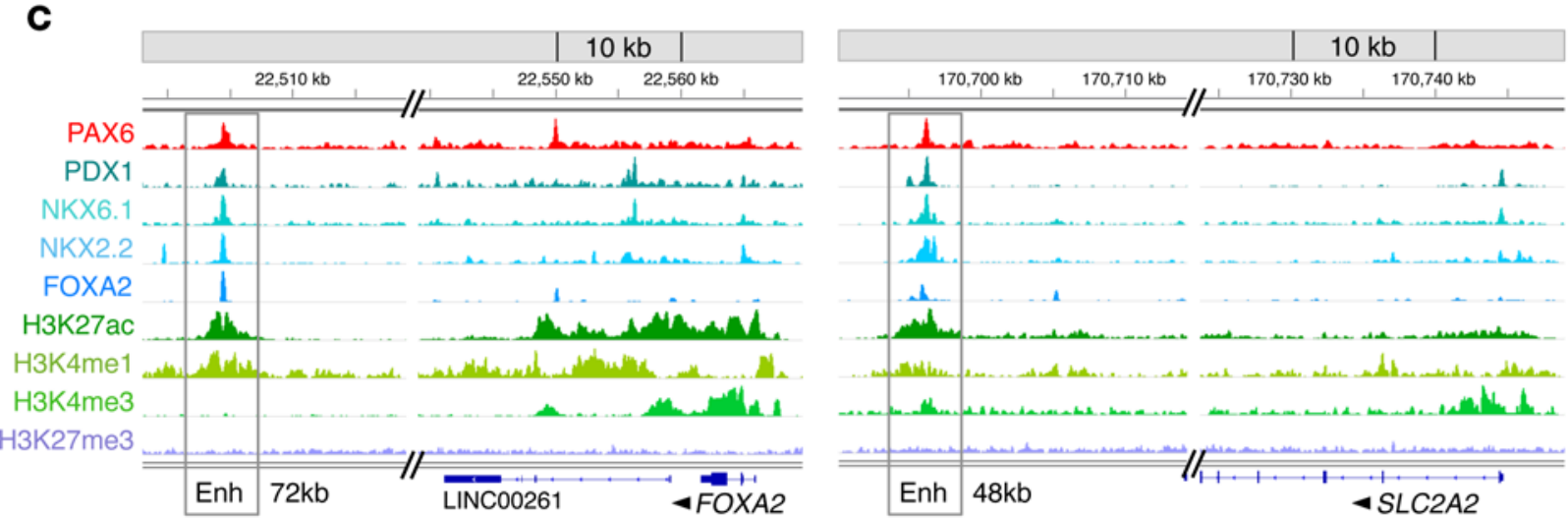

Figure 8. PAX6 function in human $\beta$ cells. (A) Change in $\beta$ cell gene expression following PAX6 knockdown in human EndoC- $\beta \mathrm{H} 2$ cells. (B) Ghrelin ${ }^{+}$cells in human islets did not express PAX6, as shown by costaining of human pancreatic sections for PAX6 (green), ghrelin (red), and insulin (blue). Material was from a 9-year-old healthy donor (obtained from nPOD). Original magnification, $\times 400$. (C) PAX6-binding sites in FOXA2 and SLC2A2 (encoding GLUT2) enhancers (Enh) (enhancers are highlighted in rectangles; the distance from the transcription start site is indicated). Note the active enhancer histone marks as well as the binding of all other $\beta$ cell TFs in the same genomic position. (D) Venn diagram showing the overlap of genes bound by PAX6 in murine and human $\beta$ cells. Note the $\beta$ cell-specific gene sets enriched in the common group (with $P$ values of $1.58 \times 10^{-21}, 1.5 \times 10^{-16}, 6.05 \times 10^{-14}, 6.65 \times 10^{-12}$, and $5.07 \times 10^{-10}$, respectively). MODY, maturity-onset diabetes of the young; T2D, type 2 diabetes.

steady-state mRNA levels of the CreER and insulin mRNAs are likely the result of very different rates of mRNA degradation, as the insulin mRNA has a half-life in excess of 3 days in rodent $\beta$ cells (58). We also examined the expression dynamics of $M a f a$, a direct activator of insulin transcription and a known target of PAX6 (59). The reduction in Mafa mRNA was rapid and occurred maximally already 1 week after tamoxifen administration, as with the kinetics of Pax 6 loss. These results suggest that Pax 6 deletion in $\beta$ cells shuts off insulin transcription rapidly and completely.

PAX6 can activate insulin transcription indirectly, through other TFs such as MAFA, or directly, by binding to the insulin gene promoter. The ChIP-seq data revealed that PAX6 binds directly to the insulin promoter (Figure 6C), which was also validated by qRT-PCR (Figure 7E), consistent with previous reports $(26,31)$. These results indicate that PAX6 is an essential positive regulator of insulin gene transcription, acting directly, by binding to the insulin gene promoter, as well as indirectly, by activating MAFA and potentially other insulin TFs.

PAX6 expression and function in human $\beta$ cells. To evaluate the role of PAX6 in human cells, we suppressed PAX6 levels in human EndoC- $\beta \mathrm{H} 2$ cells (60) using retroviral delivery of PAX6-specific siRNA. Reducing PAX6 expression in these cells yielded transcriptional changes similar to those observed in mouse $\beta$ cells. In particular, ghrelin and NEUROG3 were upregulated, while insulin, G6PC2, and other $\beta$ cell TFs were downregulated (Figure 8A). Unlike in adult mouse $\beta$ cells, glucagon mRNA was downregulated in EndoC- $\beta \mathrm{H} 2$ cells. Consistent with ghrelin upregulation upon PAX6 silencing, immunostaining of human pancreatic sections showed that PAX6 was expressed in all islet cells except for ghrelin cells, suggesting that PAX6 represses ghrelin expression in human islets, as it does in murine islets (Figure $8 \mathrm{~B}$ ).

To determine the genomic binding locations of PAX6 in human $\beta$ cells, we performed ChIP-seq on EndoC- $\beta$ H 2 cells (Figure 8C and Supplemental Table 6). By overlapping PAX6-bound sites with previously published data on ChIP-seq of histone modifications and $\beta$ cell TFs $(14,61)$, we identified distal regulatory elements. Importantly, some of these elements, such as those associated with FOXA2 and SLC2A2, are conserved between mice and humans (Figure 8C). Of 5,606 genes annotated to EndoC PAX6 ChIP-seq peaks, 2,328 (41.5\%) are also bound by PAX6 in mouse Min6 cells. Strikingly, this group of genes commonly bound by PAX6 in mice and humans is highly enriched for $\beta$ cell function genes such as PDX1, NKX6.1, HNF1A, and FOXA2 (Figure 8D and Supplemental Table 6). These findings suggest that the functions of PAX6 as an activator and repressor of genes in adult $\beta$ cells are conserved from mice to humans. 


\section{Discussion}

Our experiments show that PAX6 expression is reduced in $\beta$ cells of hyperglycemic and insulin-resistant mice and demonstrate the importance of proper expression levels of PAX6 for the maintenance of $\beta$ cell identity and glucose homeostasis. PAX6 emerges as a key regulator of the differentiated state of $\beta$ cells, with additional non-cell-autonomous effects on other islet cells, and as a novel candidate effector of $\beta$ cell failure under metabolic stress. The molecular functions of PAX6 appear to be conserved from mice to humans.

Pax 6 and transcriptional regulation of $\beta$ cell identity. We found that PAX6 is needed for maintaining the proper expression of $\beta$ cell genes as well as for repressing alternative islet cell genes, most notably hormones and transcriptional regulators of other islet cell types. This adds to the growing recognition that adult islet cells maintain a considerable degree of plasticity within the islet endocrine lineage $(4-7,15,16)$. In fact, the promiscuous expression of genes typical of multiple islet cell types appears to be the default of $\beta$ cells, and PAX6 activity is required to repress this default; this is most clearly seen in the case of ghrelin, a fetal islet hormone typically shut off in adult $\beta$ cells that is dramatically upregulated in $\beta$ cells lacking PAX6. Conceptually similar findings were reported for PDX1 (needed to prevent expression of glucagon in adult $\beta$ cells) (5) and NKX6.1 (needed to prevent somatostatin expression in adult $\beta$ cells) (6). We speculate that islet cells have a stable "ground state," which includes expression of the generic endocrine machinery, as well as low-level expression of markers of multiple islet cell types (including different hormone genes). TFs such as PAX6 sharpen this generic program into a mature endocrine cell type, with high expression of relevant genes and repression of irrelevant genes. Repression of Neurog3, a key regulator of the generic endocrine identity during development (which is derepressed in PAX6-deficient $\beta$ cells), could be involved in the progression from a ground endocrine state to a mature, fully differentiated islet cell. More experiments are needed to test this idea, which could be relevant for efforts to drive stem cell-derived endocrine cells into functional $\beta$ cells.

In $\beta$ cells, PAX6 is typically described as a transcriptional activator $(26,31)$, although studies in other tissues and cell lines have shown that it can also act as a direct repressor $(47,49,50)$. Our results from RNA-seq, ChIP-seq, and luciferase reporter experiments provide strong evidence that PAX6 acts simultaneously as a direct repressor and activator of transcription in adult $\beta$ cells via DNA elements in promoters, enhancers, and silencers. More work is needed to understand what determines whether a particular gene will be repressed or activated in $\beta$ cells by PAX 6 binding. Moreover, PAX6 is ubiquitously expressed in hormone-producing islet cells, and in each islet cell type, it is required for maintaining a particular identity, sometimes by controlling opposing genes and pathways in different cell types $(26,32)$. The differential effects of PAX6 in different islet cell types likely involve cooperation with cell-type-specific factors or coregulators. In the case of $\beta$ cells, we identified a striking resemblance between the genes controlled by PAX6 and the genes regulated by PDX1, MAFA, and NKX6.1. The similarity in the transcriptomes of mutants is at least in part driven by the fact that PAX6 controls the expression of all these TFs; we speculate that, in addition, PAX6 cooperates with these TFs to directly control the expression of target genes. Indeed, PAX6bound sites contain DNA motifs recognized by these TFs and are physically bound by these factors.

PAX6 in glucose homeostasis and diabetes. Our finding that PAX6 levels are reduced in islets of diabetic $d b / d b$ mice as well as in WT mice rendered hyperglycemic by an insulin receptor antagonist suggests that metabolic downregulation of PAX6 expression may play a role in the pathogenesis of diabetes. Previous studies reported a modest downregulation of PAX6 in $\beta$ cells cultured in high glucose (62) and in $\beta$ cells exposed to oxidative stress (7). However, in our hands, a 2-day treatment of murine and human islets with $25 \mathrm{mM}$ glucose or palmitate failed to reduce Pax6 mRNA levels (data not shown), suggesting a more complex mechanism underlying the regulation of expression in hyperglycemia in vivo. Haploinsufficiency of PAX6 in humans does interfere with $\beta$ cell function and leads to abnormal glucose homeostasis, suggesting that even partial effects on PAX6 expression may have glycemic consequences $(27,28)$. We note, however, that in expression profiles obtained from type 2 diabetic donors using both whole islets and single $\beta$ cells, no significant reduction in PAX6 expression levels was observed compared with levels detected in nondiabetic donors $(7,63,64)$. This may be attributed to euglycemia in those patients. More work is required to definitely determine whether PAX6 expression is altered in human diabetes.

How can reduced PAX6 expression contribute to diabetes? Our findings suggest several potential mechanisms. First, Pax6 deletion causes a massive loss of $\beta$ cell identity (potentially in addition to increased $\beta$ cell death), leading to hypoinsulinemia and hyperglycemia. Second, ghrelin secretion from mutant $\beta$ cells may further inhibit insulin secretion from remaining functional $\beta$ cells via mechanisms proposed elsewhere (65-67). We note, however, that thus far, there is no evidence for expression of ghrelin in islets of human patients with diabetes. Third, the non-cellautonomous expansion of somatostatin-expressing cells may further reduce insulin secretion (68). Fourth, the expansion of $\alpha$ cells may contribute to a worsening of hyperglycemia and to the unique metabolic feature of $\beta$ cell PAX6 deficiency, namely severe ketosis.

The latter possibility may relate to the emerging view that the pathogenesis of diabetes involves simultaneous $\beta$ and $\alpha$ cell dysfunction $(69,70)$ and that hypersecretion of glucagon is a key driver of diabetic symptoms (71). It has been difficult to determine the cause of $\alpha$ cell hyperfunction in diabetes. $\alpha$ Cell hyperfunction could result from a non-cell-autonomous effect of defective $\beta$ cells (71) or from reprogramming of $\beta$ cells into glucagon-expressing cells (8). Diabetes in the $\beta$ PAX6 model includes key features resulting from abnormal function of $\alpha$ cells (i.e., hyperglucagonemia and the resulting ketosis). Given the nature of this genetic model, the source of the defect has to be the $\beta$ cell. Moreover, the use of genetic lineage tracing showed that, in this case, the $\beta$ cell defect was affecting $\alpha$ cells via a non-cell-autonomous mechanism. It will be interesting to determine why PAX6 deficiency and some types of diabetes (e.g., human type 1 diabetes), but not other insults to $\beta$ cells (e.g., DTA-mediated $\beta$ cell killing) (36), induce neighboring $\alpha$ cells to expand and hypersecrete glucagon to produce ketones.

Regardless of the mechanism leading to $\alpha$ cell dysfunction, the phenotype of mice deficient for PAX6 in $\beta$ cells raises the possibility that there might be different types of $\beta$ cell failure in diabetes, 
resulting from different molecular miswirings of the $\beta$ cell, which may trigger distinct aspects of diabetes. Clinically, a wide range of diabetic phenotypes is observed (e.g., including or not including ketoacidosis, with or without severe hyperglycemia). We propose that these phenotypes do not reflect a simple graded scale of severity (modulated by peripheral tissue biology), but rather distinct molecular types of $\beta$ cell failure.

In conclusion, our work, together with recent studies $(32,33)$, highlights PAX6 as a key transcriptional regulator of adult $\beta$ cell identity and function and suggests that reduced PAX6 expression may contribute to $\beta$ cell failure in diabetes.

\section{Methods}

Mice. The mouse strains used in this study were $\operatorname{Pax}^{f / f l l}$ (72), MIPCreER (73), and Rosa26-LSL-EYFP (all from The Jackson Laboratory). Tamoxifen $(20 \mathrm{mg} / \mathrm{ml}$ in corn oil; Sigma-Aldrich) was injected s.c. into 1- to 2-month-old adult mice. Two daily doses of $8 \mathrm{mg}$ were used to achieve near-total deletion of Pax 6 in $\beta$ cells. PAX6-deficient mice are referred to herein as $\beta$ PAX 6 mice. Littermate controls are referred to herein as flox/flox (for Pax $6^{f / l l}$ Rosa26-LSL-EYFP) or $\beta Y F P$ (for MIPCreER Rosa-LSL-EYFP) mice.

$d b / d b$ mice (purchased from Envigo) and their C57BL/6J control littermates were used at the age of 3 months. Only hyperglycemic (blood glucose $>300 \mathrm{mg} / \mathrm{dl}$ ) $d b / d b$ mice were used for the experiments.

Measurements of blood glucose and plasma insulin levels were performed as described elsewhere (36). Plasma glucagon was measured with the Crystal Chem Glucagon ELISA Kit. Ketone bodies were measured with FreeStyle Optium $\beta$ Ketone Test Strips.

Immunostaining. Paraffin sections $(5-\mu \mathrm{m}$-thick) were prepared from formalin-fixed, paraffin-embedded pancreata. Sections were rehydrated, and antigen retrieval was performed. A list of the primary antibodies used and their details are provided in Supplemental Table 7. Secondary antibodies were conjugated to CY2 (1:200), CY3 (1:500), or CY5 (1:500; Jackson ImmunoResearch). Immunofluorescence images were captured using a Nikon C2 confocal microscope.

Isolation of islets of Langerhans. Islets were isolated using collagenase P (Roche) injected into the pancreatic duct, followed by Histopaque gradient (1119 and 1077; Sigma-Aldrich). Dissociation into single cells was performed by standard trypsinization.

Flow cytometry. For FACS analysis, dissociated islet cells were fixed and stained by the Cytofix/Cytoperm method (BD Biosciences). Cells were stained with guinea pig anti-insulin (1:500; Dako, Agilent Technologies) and rabbit anti-PAX6 (1:200; EMD Millipore), followed by secondary antibodies (Cy2 or Cy5 conjugated; Jackson ImmunoResearch).

Transmission electron microscopy. Pancreas was fixed with $4 \%$ paraformaldehyde and 2.5\% glutaraldehyde (Electron Microscopy Sciences [EMS]), post fixed with $1 \%$ osmium tetroxide (Sigma-Aldrich), and dehydrated with increasing concentrations of ethanol, followed by propylene oxide (Sigma-Aldrich). For embedment, we used Agar 100 Resin (Agar Scientific). For imaging, we used 80-nm sections stained with $5 \%$ uranyl acetate for 10 minutes, followed by 10 minutes with lead citrate. Samples were visualized with a JEM-1400 Plus transmission electron microscope (Jeol) equipped with a Gatan CCD camera.

$R N A$. RNA was isolated and purified from sorted $\mathrm{YFP}^{+} \beta$ cells with TRI Reagent (Sigma-Aldrich) and an RNeasy Micro Kit (QIAGEN). For gene expression profiling, RNA-seq libraries were prepared and sequenced as previously described (14).
Gene set enrichment analysis based on the hypergeometric test was performed using Genomica Software (http://genomica.weizmann. ac.il/). Gene sets with a $P$ value below 0.01 and an FDR below 0.05 were considered significant. Gene sets were from Gene Ontology (GO) or compiled from the literature as described in the text.

For qPCR assays, cDNA was synthesized using 1 ng RNA and a High-Capacity cDNA Reverse Transcription Kit (Applied Biosystems). For qRT-PCR, we used SYBR Green Mix (Quanta Biosciences) and the primers listed in Supplemental Table 7. Reactions were performed in triplicate in 96-well plates using a CFX96 Real-Time System (Bio-Rad).

Luciferase reporter assays. PAX6-bound genomic regions (140-200 bp around the peak center; see the primer sequences listed in Supplemental Table 7) were cloned into a pGL4.23 luciferase reporter plasmid upstream of a minimal promoter and transiently transfected into Min 6 cells. Luciferase activity was normalized against the activity of a cotransfected Renilla construct, and the mean values \pm SEM are shown relative to empty vector.

ChIP. Chromatin was obtained from mouse Min6 cells (cultured in DMEM with 15\% FCS and $25 \mathrm{mM}$ glucose) and from human EndoC- $\beta \mathrm{H} 2$ cells (cultured as previously described) (60). Chromatin was prepared and immunoprecipitated with rabbit anti-PAX6 (BioLegend). ChIP-seq libraries were prepared and sequenced as previously described (14). Enrichment of the mouse insulin promoter fragments was also tested by qPCR with the following primers: forward, 5'-CTGGGGAATGATGTGGAAAAT-3' and reverse, 5'-GACCAGATGGCCTGATGAAC-3'. DNA motif enrichment was performed using HOMER (74). Integrative Genomics Viewer (IGV) software was used for visualization of PAX6 peaks and additional epigenomic data (75). For integration of PAX6 genomic binding sites with whole-genome histone modifications and TF maps, we overlapped PAX6-bound sites revealed by ChIP-seq analysis of Min6 and EndoC-H2 cells with previously published ChIP-seq data for mouse and human islets or sorted $\beta$ cells, including the histone modifications H3K4me1, H3K4me3, H3K27me3, H3K27ac, and H3K9ac and the $\beta$ cell TFs PDX1, NKX6.1, FOXA2, MAFA, MAFB, NEUROD1, and NKX2.2. We used an overlap of $1 \mathrm{bp}$. For histone modifications, wings of $400 \mathrm{bp}$ were added to PAX6-bound sites.

Pax6 gene suppression. Proliferating EndoC- $\beta \mathrm{H} 2$ cells were infected with a lentivirus expressing Cre (pTRIP $\triangle$ U3-CMV-nlsCre) (60). Two weeks after Cre infection, cells were infected with a pLKO.1Puro-shPAX6 plasmid carrying the targeting sequence CGTCCATCTTTGCTTGGGAAA to silence PAX6. One week after puromycin selection, RNA was extracted from the cells and used to demonstrate efficient suppression of PAX6 and changes in the expression of additional genes.

Accession numbers. The genomic data reported here were deposited in the NCBI's Gene Expression Omnibus (GEO) database (GEO GSE87530).

Statistics. Statistical analyses were performed using an unpaired 2-tailed Student's $t$ test. Data are presented as mean \pm SEM, unless otherwise indicated.

Study approval. The joint ethics committee (IACUC) of the Hebrew University and Hadassah Medical Center approved the study protocol for animal welfare. The Hebrew University is an American Association for the Accreditation of Laboratory Animal Care (AAALAC) International-accredited institute. 


\section{Author contributions}

AS, RA-P, and YD designed the study. AS, NE, DA, JZ, RA-P, KHK, BG, and YD designed the experiments. AS, DA, NE, EF, TD, MS-R, YC-T, and RA-P conducted the experiments and analyzed the data. JZ conducted the ChIP experiments. AS, DA, and YD wrote the manuscript.

\section{Acknowledgments}

We thank the Next Generation Sequencing Core at the University of Pennsylvania (Philadelphia, Pennsylvania, USA) for RNA-seq and analysis; Agnes Klochendler (Hebrew University Medical School, Jerusalem, Israel) for help with transcriptome analysis and cell sorting; Hava Glickstein (interdepartmental equipment unit, Hebrew University Medical School, Jerusalem, Israel) for excellent help with electron micro-scopic analysis; Dana Barequet (Tel Aviv University, Tel Aviv, Israel) for initial experiments on PAX6 in endocrine cells; Ran Avrahami for whole-genome data analyses; Shalev Itzkovitz and Matan Golan (Weizmann Institute of Science, Rehovot, Israel) for help with single-molecule RNA-FISH experiments; and Raphael Scharfmann (INSERM, Institut Cochin, Paris, France) for providing EndoC- $\beta \mathrm{H} 2$ cells. This work was supported by grants from the Juvenile Diabetes Research Foundation (JDRF); the Beta Cell Biology Consortium and the Human Islet Research Network of the NIH (DK104216); the Helmsley Charitable Trust; the European Research Commission (ERC consolidator grant); the Britain Israel Research and Academic Exchange Partnership (BIRAX); the Diabetes Onderzoek Nederland (DON) Foundation; and the Israel Science Foundation and I-CORE Program of The Israel Science Foundation (ISF) (41.11, to YD). Research was performed with the support of the Network for Pancreatic Organ Donors with Diabetes (nPOD), a collaborative type 1 diabetes research project sponsored by the JDRF. The Organ Procurement Organizations (OPOs) partnering with nPOD to provide research resources are listed on the nPOD/JDRF partners website (http://www.jdrfnpod.org/for-partners/npod-partners/). This work was supported in part by a grant from USAID's American Schools and Hospitals Abroad Program for the upgrading of the Hebrew University Medical School's Flow Cytometry Laboratory. RA-P was supported by the United States-Israel Binational Science Foundation (BSF; grant 2013016) and the ISF (grant 228/14). AS received fellowships from the Adams Foundation and the Ariane de Rothschild Women Doctoral Program.

Address correspondence to: Yuval Dor, The Hebrew UniversityHadassah Medical School, Jerusalem 91120, Israel. Phone: 972.2.6757181; E-mail: yuvald@ekmd.huji.ac.il. Or to: Dana Avrahami, Hadassah Medical Center, Jerusalem 91120, Israel. Phone: 972.2.6777351; E-mail: dana.tzfati@mail.huji.ac.il. Or to: Ruth Ashery-Padan, Sackler Faculty of Medicine, Tel Aviv University, Tel Aviv 69978, Israel. Phone: 972.3.6409331; E-mail: ruthash@ post.tau.ac.il.
1. Arda HE, Benitez CM, Kim SK. Gene regulatory networks governing pancreas development. Dev Cell. 2013;25(1):5-13.

2. Zaret KS. Genetic programming of liver and pancreas progenitors: lessons for stem-cell differentiation. Nat Rev Genet. 2008;9(5):329-340.

3. Rieck S, Bankaitis ED, Wright CV. Lineage determinants in early endocrine development. Semin Cell Dev Biol. 2012;23(6):673-684.

4. Ziv O, Glaser B, Dor Y. The plastic pancreas. Dev Cell. 2013;26(1):3-7.

5. Gao T, et al. Pdx1 maintains $\beta$ cell identity and function by repressing an $\alpha$ cell program. Cell Metab. 2014;19(2):259-271.

6. Taylor BL, Liu FF, Sander M. Nkx6.1 is essential for maintaining the functional state of pancreatic beta cells. Cell Rep. 2013;4(6):1262-1275.

7. Guo S, et al. Inactivation of specific $\beta$ cell transcription factors in type 2 diabetes. J Clin Invest. 2013;123(8):3305-3316.

8. Talchai C, Xuan S, Lin HV, Sussel L, Accili D. Pancreatic $\beta$ cell dedifferentiation as a mechanism of diabetic $\beta$ cell failure. Cell. 2012;150(6):1223-1234 .

9. Piccand J, et al. Rfx6 maintains the functional identity of adult pancreatic $\beta$ cells. Cell Rep. 2014;9(6):2219-2232

10. Brereton MF, et al. Reversible changes in pancreatic islet structure and function produced by elevated blood glucose. Nat Commun. 2014;5:4639.

11. Wang Z, York NW, Nichols CG, Remedi MS. Pancreatic $\beta$ cell dedifferentiation in diabetes and redifferentiation following insulin therapy. Cell Metab. 2014;19(5):872-882.

12. Butler AE, Dhawan S. $\beta$-cell identity in type 2 diabetes: Lost or found? Diabetes. 2015;64(8):2698-2700.
13. Cinti F, et al. Evidence of $\beta$-cell dedifferentiation in human type 2 diabetes. JClin Endocrinol Metab. 2016;101(3):1044-1054.

14. Bramswig NC, et al. Epigenomic plasticity enables human pancreatic $\alpha$ to $\beta$ cell reprogramming. J Clin Invest. 2013;123(3):1275-1284.

15 . Chera $S$, et al. Diabetes recovery by age-dependent conversion of pancreatic $\delta$-cells into insulin producers. Nature. 2014;514(7523):503-507.

16. Thorel F, et al. Conversion of adult pancreatic alpha-cells to beta-cells after extreme beta-cell loss. Nature. 2010;464(7292):1149-1154.

17. Shaham O, Menuchin Y, Farhy C, Ashery-Padan R. Pax6: a multi-level regulator of ocular development. Prog Retin Eye Res. 2012;31(5):351-376.

18. Manuel MN, Mi D, Mason JO, Price DJ. Regulation of cerebral cortical neurogenesis by the Pax6 transcription factor. Front Cell Neurosci. 2015;9:70.

19. Ypsilanti AR, Rubenstein JL. Transcriptional and epigenetic mechanisms of early cortical development: An examination of how Pax6 coordinates cortical development. JComp Neurol. 2016;524(3):609-629.

20. Simpson TI, Price DJ. Pax6; a pleiotropic player in development. Bioessays. 2002;24(11):1041-1051.

21. Sun J, Rockowitz S, Xie Q, Ashery-Padan R, Zheng D, Cvekl A. Identification of in vivo DNA-binding mechanisms of Pax6 and reconstruction of Pax6-dependent gene regulatory networks during forebrain and lens development. Nucleic Acids Res. 2015;43(14):6827-6846.

22. Xie Q, et al. Pax6 interactions with chromatin and identification of its novel direct target genes in lens and forebrain. PLoS One. 2013;8(1):e54507.

23. Shaham O, et al. Pax6 regulates gene expression in the vertebrate lens through miR-204. PLoS Genet. 2013;9(3):e1003357.

24. Ashery-Padan R, et al. Conditional inactivation of Pax6 in the pancreas causes early onset of diabetes. Dev Biol. 2004;269(2):479-488.

25. St-Onge L, Sosa-Pineda B, Chowdhury K, Mansouri A, Gruss P. Pax6 is required for differentiation of glucagon-producing alpha-cells in mouse pancreas. Nature. 1997;387(6631):406-409.

26. Sander M, Neubüser A, Kalamaras J, Ee HC, Martin GR, German MS. Genetic analysis reveals that PAX6 is required for normal transcription of pancreatic hormone genes and islet development. Genes Dev. 1997;11(13):1662-1673.

27. Yasuda T, et al. PAX6 mutation as a genetic factor common to aniridia and glucose intolerance. Diabetes. 2002;51(1):224-230.

28. Ding J, et al. Pax6 haploinsufficiency causes abnormal metabolic homeostasis by down-regulating glucagon-like peptide 1 in mice. Endocrinology. 2009;150(5):2136-2144.

29. Nishi M, et al. A case of novel de novo paired box gene 6 (PAX6) mutation with early-onset diabetes mellitus and aniridia. Diabet Med. 2005;22(5):641-644.

30. Ahlqvist $\mathrm{E}$, et al. A common variant upstream of the PAX6 gene influences islet function in man. Diabetologia. 2012;55(1):94-104.

31. Gosmain Y, Katz LS, Masson MH, Cheyssac C, Poisson C, Philippe J. Pax6 is crucial for $\beta$-cell function, insulin biosynthesis, and glucose-induced insulin secretion. Mol Endocrinol. 2012;26(4):696-709.

32. Hart AW, Mella S, Mendrychowski J, van Heyningen V, Kleinjan DA. The developmental regulator Pax6 is essential for maintenance of islet cell 
function in the adult mouse pancreas. PLoS One. 2013;8(1):e54173.

33. Ahmad Z, Rafeeq M, Collombat P, Mansouri A. Pax6 inactivation in the adult pancreas reveals ghrelin as endocrine cell maturation marker. PLoS One. 2015;10(12):e0144597.

34. Peter NM, Leyland M, Mudhar HS, Lowndes J, Owen KR, Stewart H. PAX6 mutation in association with ptosis, cataract, iris hypoplasia, corneal opacification and diabetes: a new variant of familial aniridia? Clin Experiment Ophthalmol. 2013;41(9):835-841.

35. Schäffer L, et al. A novel high-affinity peptide antagonist to the insulin receptor. Biochem Biophys Res Commun. 2008;376(2):380-383.

36. Nir T, Melton DA, Dor Y. Recovery from diabetes in mice by beta cell regeneration. J Clin Invest. 2007;117(9):2553-2561.

37. Porat $S$, et al. Control of pancreatic $\beta$ cell regeneration by glucose metabolism. Cell Metab. 2011;13(4):440-449.

38. Quant PA, Tubbs PK, Brand MD. Treatment of rats with glucagon or mannoheptulose increases mitochondrial 3-hydroxy-3-methylglutaryl-CoA synthase activity and decreases succinyl-CoA content in liver. Biochem J. 1989;262(1):159-164.

39. Foster DW, McGarry JD. The regulation of ketogenesis. Ciba Found Symp. 1982;87:120-131.

40. Laffel L. Ketone bodies: a review of physiology, pathophysiology and application of monitoring to diabetes. Diabetes Metab Res Rev. 1999;15(6):412-426

41. Kojima M, Kangawa K. Ghrelin: structure and function. Physiol Rev. 2005;85(2):495-522.

42. Zhang J, McKenna LB, Bogue CW, Kaestner $\mathrm{KH}$. The diabetes gene Hhex maintains $\delta$-cell differentiation and islet function. Genes Dev. 2014;28(8):829-834.

43. Sachdeva MM, et al. Pdx1 (MODY4) regulates pancreatic beta cell susceptibility to ER stress. Proc Natl Acad Sci U S A. 2009;106(45):19090-19095.

44. Hang $Y$, et al. The MafA transcription factor becomes essential to islet $\beta$-cells soon after birth. Diabetes. 2014;63(6):1994-2005.

45. Anderson KR, White P, Kaestner KH, Sussel L. Identification of known and novel pancreas genes expressed downstream of $\mathrm{Nkx} 2.2$ during development. BMC Dev Biol. 2009;9:65.

46. Prado CL, Pugh-Bernard AE, Elghazi L, SosaPineda B, Sussel L. Ghrelin cells replace insulinproducing beta cells in two mouse models of pancreas development. Proc Natl Acad Sci U S A. 2004;101(9):2924-2929.

47. Xie Q, Ung D, Khafizov K, Fiser A, Cvekl A. Gene regulation by PAX6: structural-functional correlations of missense mutants and transcriptional control of Trpm3/miR-204. Mol Vis. 2014;20:270-282.
48. Epstein JA, Glaser T, Cai J, Jepeal L, Walton DS, Maas RL. Two independent and interactive DNA-binding subdomains of the Pax6 paired domain are regulated by alternative splicing. Genes Dev. 1994;8(17):2022-2034.

49. Duncan MK, Haynes JI, Cvekl A, Piatigorsky J. Dual roles for Pax-6: a transcriptional repressor of lens fiber cell-specific beta-crystallin genes. Mol Cell Biol. 1998;18(9):5579-5586.

50. Zhang X, et al. Pax6 is a human neuroectoderm cell fate determinant. Cell Stem Cell. 2010;7(1):90-100.

51. Epstein J, Cai J, Glaser T, Jepeal L, Maas R. Identification of a Pax paired domain recognition sequence and evidence for DNAdependent conformational changes. J Biol Chem. 1994;269(11):8355-8361.

52. Avrahami D, et al. Aging-dependent demethylation of regulatory elements correlates with chromatin state and improved $\beta$ cell function. Cell Metab. 2015;22(4):619-632.

53. Tennant BR, et al. Identification and analysis of murine pancreatic islet enhancers. Diabetologia. 2013;56(3):542-552.

54. Lynn FC, Smith SB, Wilson ME, Yang KY, Nekrep $\mathrm{N}$, German MS. Sox 9 coordinates a transcriptional network in pancreatic progenitor cells. Proc Natl Acad Sci U S A. 2007;104(25):10500-10505.

55. Lee JC, et al. Regulation of the pancreatic pro-endocrine gene neurogenin3. Diabetes. 2001;50(5):928-936.

56. Liu T, et al. Pax6 directly down-regulates Pcsk1n expression thereby regulating $\mathrm{PC} 1 / 3$ dependent proinsulin processing. PLoS One. 2012;7(10):e46934.

57. Wen JH, et al. Paired box 6 (PAX6) regulates glucose metabolism via proinsulin processing mediated by prohormone convertase $1 / 3(\mathrm{PC} 1 / 3)$. Diabetologia. 2009;52(3):504-513.

58. Welsh M, Nielsen DA, MacKrell AJ, Steiner DF. Control of insulin gene expression in pancreatic beta-cells and in an insulin-producing cell line, RIN-5F cells. II. Regulation of insulin mRNA stability. J Biol Chem. 1985;260(25):13590-13594.

59. Raum JC, et al. Islet beta-cell-specific MafA transcription requires the 5 '-flanking conserved region 3 control domain. Mol Cell Biol. 2010;30(17):4234-4244.

60. Scharfmann R, et al. Development of a conditionally immortalized human pancreatic $\beta$ cell line. JClin Invest. 2014;124(5):2087-2098.

61. Pasquali L, et al. Pancreatic islet enhancer clusters enriched in type 2 diabetes risk-associated variants. Nat Genet. 2014;46(2):136-143.

62. Balakrishnan S, Sadasivam M, Kannan A, Panneerselvam A, Prahalathan C. Glucose modulates Pax6 expression through the JNK/p38 MAP kinase pathway in pancreatic beta-cells. Life Sci.
2014;109(1):1-7.

63. Wang YJ, et al. Single-cell transcriptomics of the human endocrine pancreas. Diabetes. 2016;65(10):3028-3038.

64. Fadista J, et al. Global genomic and transcriptomic analysis of human pancreatic islets reveals novel genes influencing glucose metabolism. Proc Natl Acad Sci U S A. 2014;111(38):13924-13929.

65. Heppner KM, Tong J. Mechanisms in endocrinology: regulation of glucose metabolism by the ghrelin system: multiple players and multiple actions. Eur JEndocrinol. 2014;171(1):R21-R32.

66. Adriaenssens AE, et al. Transcriptomic profiling of pancreatic alpha, beta and delta cell populations identifies delta cells as a principal target for ghrelin in mouse islets. Diabetologia. 2016;59(10):2156-2165.

67. DiGruccio MR, et al. Comprehensive alpha, beta and delta cell transcriptomes reveal that ghrelin selectively activates delta cells and promotes somatostatin release from pancreatic islets. $\mathrm{Mol}$ Metab. 2016;5(7):449-458.

68. Rutter GA. Regulating glucagon secretion: somatostatin in the spotlight. Diabetes. 2009;58(2):299-301.

69. Lee $Y$, et al. Metabolic manifestations of insulin deficiency do not occur without glucagon action. Proc Natl Acad Sci U S A 2012;109(37):14972-14976.

70. Lee Y, Wang MY, Du XQ, Charron MJ, Unger RH. Glucagon receptor knockout prevents insulin-deficient type 1 diabetes in mice. Diabetes. 2011;60(2):391-397.

71. Unger RH, Cherrington AD. Glucagonocentric restructuring of diabetes: a pathophysiologic and therapeutic makeover. JClin Invest. 2012;122(1):4-12.

72. Ashery-Padan R, Marquardt T, Zhou X, Gruss P. Pax6 activity in the lens primordium is required for lens formation and for correct placement of a single retina in the eye. Genes Dev. 2000;14(21):2701-2711

73. Tamarina NA, Roe MW, Philipson L. Characterization of mice expressing Ins1 gene promoter driven CreERT recombinase for conditional gene deletion in pancreatic $\beta$-cells. Islets. 2014;6(1):e27685.

74. Heinz S, et al. Simple combinations of lineagedetermining transcription factors prime cisregulatory elements required for macrophage and B cell identities. Mol Cell.2010;38(4):576-589.

75. Robinson JT, et al. Integrative Genomics Viewer. Nat Biotechnol. 2011;29(1):24-26.

76. Blum B, Hrvatin SS, Schuetz C, Bonal C, Rezania A, Melton DA. Functional beta-cell maturation is marked by an increased glucose threshold and by expression of urocortin 3. Nat Biotechnol. 2012;30(3):261-264. 